\title{
Caspase-3-dependent export of TCTP: a novel pathway for antiapoptotic intercellular communication
}

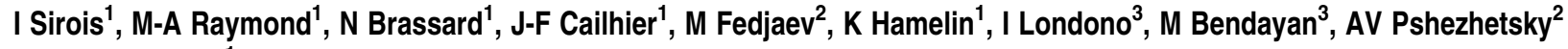 \\ and M-J Hébert ${ }^{\star 1}$
}

The apoptotic program incorporates a paracrine component of importance in fostering tissue repair at sites of apoptotic cell deletion. As this paracrine pathway likely bears special importance in maladaptive intercellular communication leading to vascular remodeling, we aimed at further defining the mediators produced by apoptotic endothelial cells (EC), using comparative and functional proteomics. Apoptotic EC were found to release nanovesicles displaying ultrastructural characteristics, protein markers and functional activity that differed from apoptotic blebs. Tumor susceptibility gene 101 and translationally controlled tumor protein (TCTP) were identified in nanovesicle fractions purified from medium conditioned by apoptotic EC and absent from purified apoptotic blebs. Immunogold labeling identified TCTP on the surface of nanovesicles purified from medium conditioned by apoptotic EC and within multivesicular blebs in apoptotic EC. These nanovesicles induced an extracellular signal-regulated kinases 1/2 (ERK 1/2)-dependent antiapoptotic phenotype in vascular smooth muscle cells (VSMC), whereas apoptotic blebs did not display antiapoptotic activity on VSMC. Caspase-3 biochemical inhibition and caspase-3 RNA interference in EC submitted to a proapoptotic stimulus inhibited the release of nanovesicles. Also, TCTP siRNAs in EC attenuated the antiapoptotic activity of purified nanovesicles on VSMC. Collectively, these results identify TCTP-bearing nanovesicles as a novel component of the paracrine apoptotic program of potential importance in vascular repair.

Cell Death and Differentiation (2011) 18, 549-562; doi:10.1038/cdd.2010.126; published online 22 October 2010

Although classically considered a 'silent' process, apoptotic cells are known to actively release paracrine mediators that, in turn, control the phagocytosis of apoptotic cells. For example, apoptotic cells release lysophosphatidylcholine, which favors the recruitment of professional phagocytes and the clearance of apoptotic cells. ${ }^{1}$ They also secrete lactoferrin as a means of promoting the migration of mononuclear leukocytes, while inhibiting the migration of polymorphonuclear leukocytes. ${ }^{2}$ Recent results suggest that this paracrine response also aims at fostering tissue remodeling and repair. A C-terminal fragment of perlecan (LG3), released as a consequence of cathepsin $L$ translocation from apoptotic endothelial cells (EC), induces Bcl-xl upregulation and resistance to apoptosis in fibroblasts, mesenchymal stem cells and smooth muscle cells, all of which are pivotal to tissue or vascular remodeling and repair. ${ }^{3-7}$ Recently, we showed that caspase-3 activation in EC favors the release of connective tissue growth factor (CTGF), ${ }^{8}$ which induces the differentiation of fibroblasts into myofibroblasts, a mandatory process in all forms of repair. To date, caspase- 3 activation has been implicated in the release of most, if not all, mediators secreted by apoptotic cells, ${ }^{1,2,6,8}$ suggesting that the paracrine response is embedded within the effector phase of apoptosis.

Dysregulation of apoptosis within cellular constituents of the vessel wall is pivotal to most forms of pathological vascular remodeling. Chronic transplant vasculopathy, systemic sclerosis and classical atherosclerosis are all characterized by the enhanced apoptosis of EC and decreased apoptosis of neointimal cells. ${ }^{9,10}$ Neointima formation develops initially through the acquisition of a synthetic and antiapoptotic phenotype in neointimal cells associated with extracellular signal-regulated kinases $1 / 2$ (ERK 1/2) activation and $\mathrm{Bcl}-\mathrm{xl}$ overexpression. ${ }^{11-13}$ Mediators produced by apoptotic EC, such as LG3 and CTGF, actively induce ERK 1/2and/or Bcl-xl-dependent antiapoptotic phenotypes in vascular smooth muscle cells (VSMC), mesenchymal stem cells and myofibroblasts. ${ }^{3-8}$ This raises the possibility that a chronic

\footnotetext{
${ }^{1}$ Research Centre, Centre hospitalier de l'Université de Montréal (CRCHUM), 1560 rue Sherbrooke Est, Montréal, Quebec, Canada; ${ }^{2}$ Research Centre, CHU SainteJustine, 3175 Côte Sainte-Catherine, Montréal, Quebec, Canada and ${ }^{3}$ Department of Pathology and Cell Biology, Faculty of Medicine, Université de Montréal, 2900 boul. Edouard-Montpetit, R-810, C.P. 6128 Succ. Centre-ville, Montréal, Quebec, Canada

*Corresponding author: M-J Hébert, Research Centre, Centre hospitalier de l'Universite de Montreal, 1560 rue Sherbrooke East, Montréal, QC H2L 4M1, Canada. Tel: (514) 890-8000 Ext. 25393; Fax: (514) 412-7624; E-mail: marie-josee.hebert.chum @ssss.gouv.qc.ca

Keywords: apoptosis; exosomes; nanovesicles; apoptotic blebs

Abbreviations: 2D-LC-MS/MS, two-dimensional liquid chromatography and tandem mass spectrometry; CTGF, connective tissue growth factor; DMSO, dimethylsulfoxide; EC, endothelial cells; ERK 1/2, extracellular signal-regulated kinases 1/2; FPLC, fast protein liquid chromatography; HO, Hoechst 33342 (2'-(4ethoxyphenyl)-5-(4-methyl-1-piperazinyl)-2.5'-bi-1H-benzimidazole); HUVEC, human umbilical vascular endothelial cells; LDH, lactate dehydrogenase; LG3, C-terminal fragment of perlecan; MMC, mitomycin C; MVB, multivesicular bodies; PARP, poly(ADP-ribose) polymerase; PI, propidium iodide; SDS-PAGE, sodium dodecyl sulfatepolyacrylamide gel electrophoresis; siRNAs, small interfering RNAs; SN, exosome-free supernatant; SS, serum starvation; SSC, serum-free media conditioned by endothelial cells; TCTP, translationally-controlled tumor protein; TSG 101, tumor susceptibility gene 101; VSMC, vascular smooth muscle cells; LC3, microtubuleassociated proteins $1 \mathrm{~A}$ and $1 \mathrm{~B}$, light chain 3 ; mTOR, mammalian target of rapamycin
}

Received 17.12.09; revised 04.8.10; accepted 13.8.10; Edited by P Vandenabeele; published online 22.10.10 
increase in EC apoptosis, resulting in a sustained paracrine response, could contribute to maladaptive vascular repair.

Here, we aim at further characterizing the molecular paracrine signals released by apoptotic EC, with a specific focus on proteins of importance in fostering a neointimal phenotype in VSMC. We used comparative and functional proteomics to identify novel antiapoptotic mediators released by apoptotic EC through caspase-dependent pathways.

\section{Results}

Serum starvation induces a pure apoptotic response in EC. To study the secretome of apoptotic EC, human umbilical vein endothelial cells (HUVEC) were rendered apoptotic in vitro by serum starvation (SS) for $4 \mathrm{~h}$, as reported previously. ${ }^{3-8}$ In serum-starved EC, the percentage of cells with chromatin condensation in absence of cell membrane permeabilization increased over time, suggesting increased apoptosis (Figure 1a). The absence of lactate dehydrogenase $(\mathrm{LDH})$ release in serum-starved EC was also consistent with absence of cell membrane permeabilization (Figure 1b). Loss of mitochondrial integrity was evident after $2 \mathrm{~h}$ of SS (Figure 1c), with concomitant activation of caspase9 and caspase-3 and poly(ADP-ribose) polymerase (PARP) cleavage (Figure 1d and e). Preincubating EC with the pancaspase inhibitor (ZVAD-FMK) or with inhibitors of caspase-3 and caspase-9 (DEVD-FMK and LEHD-FMK, respectively) before SS effectively blocked caspase(s) activation, PARP cleavage and chromatin condensation (Figure $1 \mathrm{~d}-\mathrm{g}$ and Supplementary Figure S2a). Cell membrane permeabilization, suggestive of necrosis, was not significantly modulated by ZVAD-FMK, DEVD-FMK or LEHD-FMK compared with the vehicle (dimethylsulfoxide, DMSO) (Figure 1g). Finally, as expected, pan-caspase inhibition in serum-starved EC did not prevent mitochondrial permeabilization (Figure 1c) and did not modulate p53 protein levels (Figure 1h). Collectively, these results demonstrate a pure intrinsic apoptotic response in serum-starved EC.

Characterization of the secretome of apoptotic EC. Serum-free media conditioned by apoptotic and nonapoptotic EC (SSC-Apo and SSC-No-Apo, respectively) were generated by exposing equal EC numbers to either vehicle (DMSO) or ZVAD-FMK for $2 \mathrm{~h}$, followed by medium change and SS for $4 \mathrm{~h}$, as described above and in Figure 2a. Proteins secreted by apoptotic EC downstream of caspase(s) activation were studied through comparison of equal amounts of proteins precipitated from equal volumes of conditioned media cleared of apoptotic blebs and confirmed by flow cytometric analysis (Figure 2b). Proteins from SSC-Apo and SSC-No-Apo were analyzed comparatively, by either two-dimensional-liquid chromatography tandem mass spectrometry (2D-LC-MS/MS) (Figure 2c) or SDSPAGE LC-MS/MS ${ }^{14}$ (Figure $2 \mathrm{~d}$ ). The latter revealed a distinct pattern of Coomassie blue protein staining in SSC-Apo compared with SSC-No-Apo. These results are in keeping with our previous work, describing increased protein secretion downstream of caspase(s) activation by apoptotic EC. ${ }^{6}$ Concomitantly, SSC-Apo was fractionated by fast protein liquid chromatography (FPLC), and the antiapoptotic activity of each fraction was evaluated on VSMC. ${ }^{3}$ The protein mediators present in fractions with significant antiapoptotic activity were further characterized by SDS-PAGE LC-MS/MS.

To be considered a specific component of the apoptotic secretome, a protein identified by multifaceted screening had to meet the following criteria: (1) it had to be identified by at least two out of the three different MS/MS approaches, (2) it had to be found exclusively in SSC-Apo and (3) it had to be of human origin. Among the 27 proteins meeting the screening criteria (Table 1), only 11 had known secretion signals and 10 had been previously reported as components of exosomal nanovesicles (Table 1). To evaluate whether proteins known to be secreted through nanovesicles were overrepresented in SSC-Apo compared with SSC-No-Apo, the proteomics data were reanalyzed in search of classical nanovesicle (exosome and exosome-like) markers identified at least once by any proteomics strategies in either SSC-Apo or SSC-No-Apo. In addition to the proteins listed in Table 1, twelve exosomal proteins were identified solely in SSC-Apo, including the two highly characteristic exosomal markers (Alix and CD63), ${ }^{15}$ whereas only two exosomal proteins were found exclusively in SSC-No-Apo (Table 2). These results suggested increased release of nanovesicles by apoptotic EC downstream of caspase(s) activation.

Caspase(s) activation in apoptotic EC fosters the release of antiapoptotic exosome-like nanovesicles. To determine whether apoptosis specifically increases the release of nanovesicle biomarkers over other proteins, levels of Grp96, an endoplasmic reticulum resident protein, and tubulin, a microtubule-associated protein, were evaluated in intracellular protein extracts and corresponding supernatants of serum-starved EC. Grp96 and tubulin intracellular protein levels were stable throughout SS (Figure 3a). Grp96 and tubulin were not recovered in medium conditioned by serumstarved apoptotic EC (Figure 3a), ruling out the possibility of unspecific protein leakage. These results are in keeping with the absence of cell membrane permeabilization in serum-starved EC (Figure 1a and b). However, protein levels of tumor susceptibility gene 101 (TSG 101) and translationally controlled tumor protein (TCTP), two classical markers of exosomal nanovesicles, ${ }^{15-18}$ increased over time in media conditioned by serum-starved EC (Figure 3a).

To specifically address the importance of caspase(s) activation in the production of exosomes or exosome-like nanovesicles by apoptotic EC, protein levels of the exosomal marker TCTP were compared in equal volumes of SSC-Apo and SSC-No-Apo conditioned by equal EC numbers (Figure 3b and Supplementary Figure S2b and S3). Elevated TCTP levels were evident in total unfractionated conditioned medium (Total) and in nanovesicle extracts (Exo) of SSC-Apo compared with SSC-No-Apo. Ponceau red staining confirmed augmented protein content in the nanovesicle fraction of SSC-Apo compared with SSC-No-Apo (Figure 3b). In nanovesicle-free supernatant (SN), the TCTP signal was recovered at similar levels in SSC-Apo and SSC-No-Apo (Figure 3b). In SSC-Apo, TCTP was detectable at different molecular weights ( $\sim 23,50$ and $75 \mathrm{kDa})$ in media conditioned by serum-starved EC (Figure $3 a-c$ ), in agreement with the 
Chromatin condensation

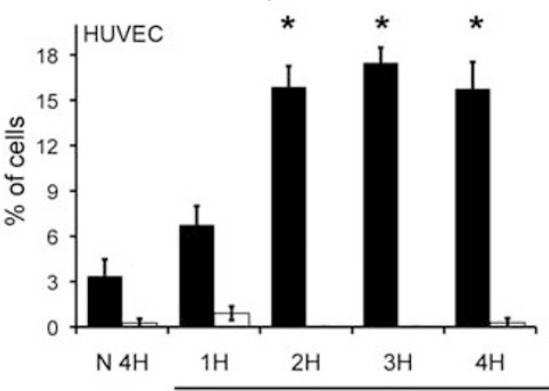

Serum starvation
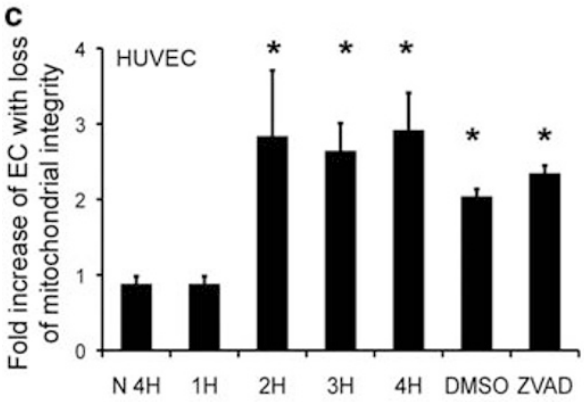

d

b

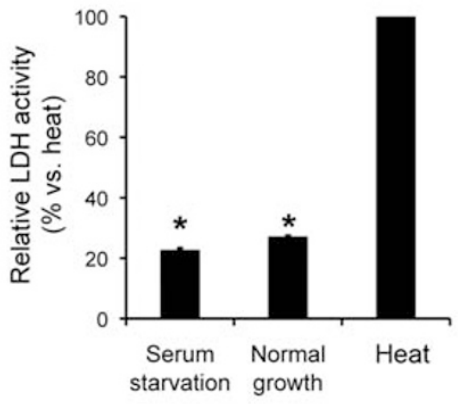

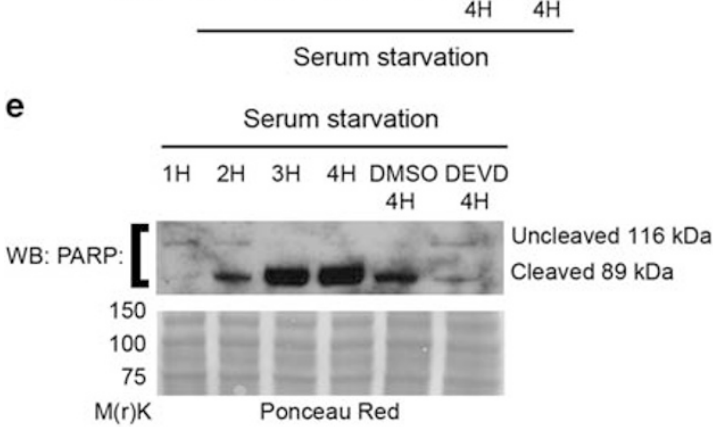

g

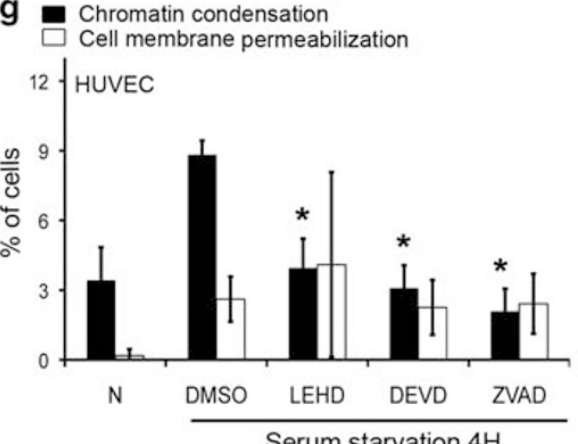

f

Serum starvation $4 \mathrm{H}$

DMSO DEVD LEHD

Active Caspase

Actin

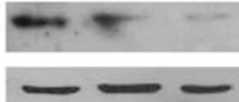
p25

LEHD DMSO DEVD

Active Caspase 3

Actin

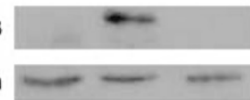

p17-19

$45 \mathrm{kDa}$

h

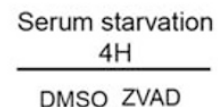

WB: $p 53$

75

50

37

$\mathrm{M}(\mathrm{r}) \mathrm{K}$ Ponceat

Red

Figure 1 Serum starvation induces a pure apoptotic response in EC. (a) Percentage of cells with increased chromatin condensation and cell membrane permeabilization (as evaluated with $\mathrm{HO}$ and PI staining) in EC exposed to normal medium (N) or serum starvation (SS) for $1-4 \mathrm{~h}$. ${ }^{*} P \leq 0.02$ versus $\mathrm{N}, n=4$. (b) LDH activity in media conditioned by normal $\mathrm{EC}$ and $\mathrm{EC}$ serum starved for $4 \mathrm{~h}$ or heated at $65^{\circ} \mathrm{C}$ for $30 \mathrm{~min}$ (positive control for cell membrane permeabilization). ${ }^{*} P<1 \times 10^{-6}$ versus heat, $n=3$. (c) JC-1 staining for evaluation of mitochondrial permeabilization in EC treated as described in (a) or preincubated with the pan-caspase inhibitor (ZVAD-FMK) $100 \mu \mathrm{M}$ or vehicle (DMSO) and serum starved for $4 \mathrm{~h} .{ }^{*} P \leq 0.02$ versus normal, $n=6$. (d) Immunoblots for active forms of caspase-9 (p25) and caspase-3 ( $p 17-\mathrm{p} 19$ ) in EC treated as in (c). Representative of five experiments. (e) Immunoblots for uncleaved and cleaved PARP in EC treated as in (c-d). Representative of two experiments. Ponceau red staining is shown as loading control. (f) Immunoblots for active forms of caspase-9 and caspase-3 in EC exposed for $4 \mathrm{~h}$ to $\mathrm{N}$ or preincubated with vehicle (DMSO), $100 \mu \mathrm{M}$ of the caspase-9 inhibitor (LEHD) or the caspase-3 inhibitor (DEVD) as in (c-d). Representative of five experiments. (g) Percentage of cells with increased chromatin condensation and cell membrane permeabilization (as evaluated with $\mathrm{HO}$ and $\mathrm{PI}$ staining) in $\mathrm{EC}$ as treated in (c). ${ }^{*} P<0.05$ versus serum starvation DMSO, $n \geq 12$. (h) Immunoblots for $p 53$ in EC as treated in (c). Representative of four experiments. Ponceau red staining is shown as loading control for (e) and (h). The color reproduction of this figure is available on the html full text version of the manuscript 
a

Vehicle (DMSO 0.5\%)

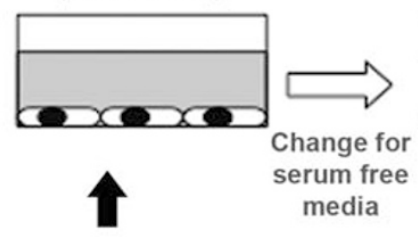

HUVEC

(25 000 cells $/ \mathrm{cm}^{2}$ )

Pre-incubation

$2 \mathrm{H}$ in normal

medium

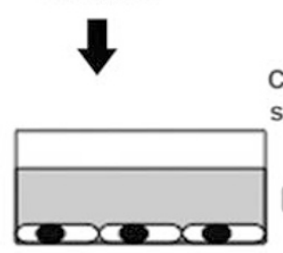

ZVAD-FMK 100 uM, (DMSO 0.5\%)

Irreversible pancaspase inhibitor
Serum-free medium conditioned by apoptotic EC

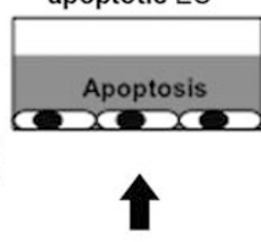

Serum starvation $4 \mathrm{H}$

Change for serum free $\longrightarrow$

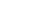

(1)

Serum-free medium conditioned by

NON-apoptotic EC

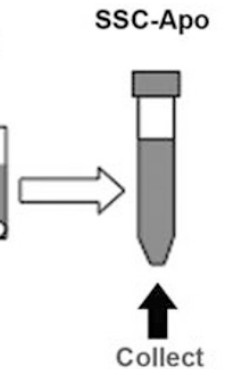

supernatant (SSC)

conditioned by

equal cell numbers

in equal volumes

of RPMI

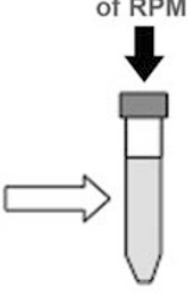

b

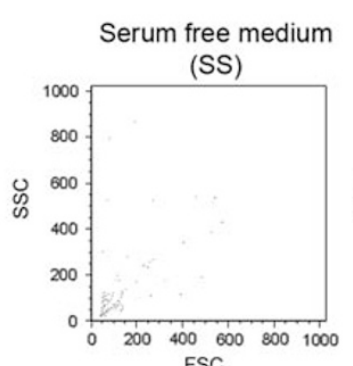

Serum free medium conditioned by apototic EC

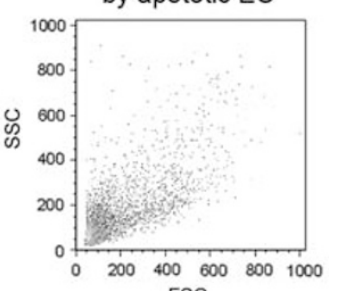

Serum free medium conditioned by apoptotic EC depleted of apoptotic blebs

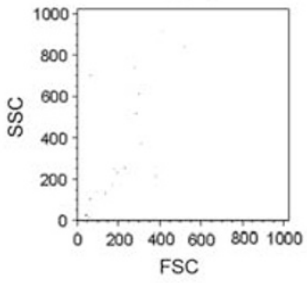

C SSC-Apo

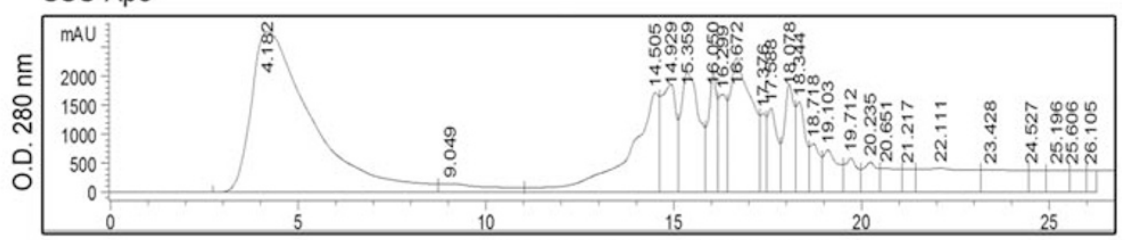

Elution time ( 1 fraction/minute)

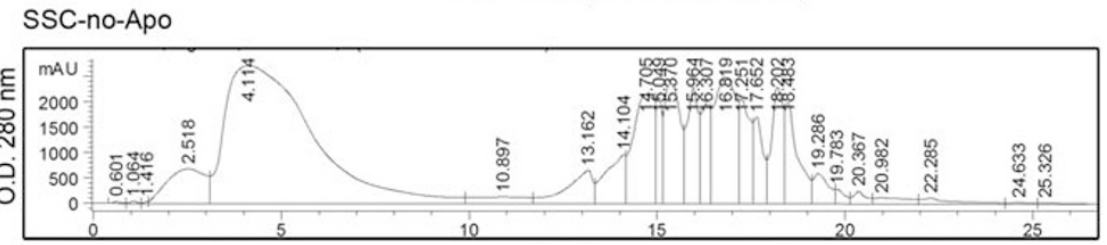

d

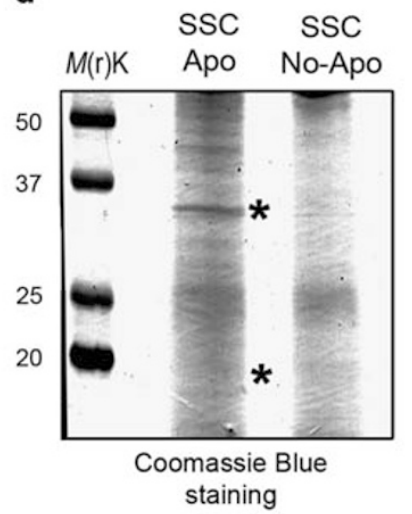

Elution time ( 1 fraction/minute)

Figure 2 Characterization of the secretome of apoptotic EC. (a) Schematic representation of the experimental strategy for generating serum-free media (conditioned by equal EC numbers in equal volumes of serum-free media) by apoptotic (SSC-Apo) and non-apoptotic EC (SSC-No-Apo). (b) Flow cytometry analysis providing evidence of depletion of apoptotic blebs before MS/MS analysis. (c) In-solution fractionation (2D-LC-MS/MS) of SSC-Apo and SSC-No-Apo generated as described in (a). Tryptic protein digests $(500 \mu \mathrm{g}$ ) from conditioned media (SSC-Apo and SSC-No-Apo) were fractionated with high-performance liquid chromatography into 25 fractions as represented by the chromatograms (O.D. $280 \mathrm{~nm}$ in function of elution time). Tryptic digests from fractions 3-22 of both samples were submitted individually to peptide sequencing by LC-MS/MS. (d) In-gel fractionation of SSC-Apo and SSC-No-Apo generated as described in (a). Serum-free conditioned media were concentrated on a 30-kDa membrane by ultrafiltration. $125 \mu \mathrm{g}$ of proteins were separated on 15\% SDS-PAGE gel and stained with Coomassie blue. The gel was cut into 20 pairs of bands, followed by in-gel trypsinization and peptide sequencing by LC-MS/MS. * represents bands corresponding to TCTP. The color reproduction of this figure is available on the html full text version of the manuscript

SDS-PAGE LC-MS/MS results where TCTP sequences were identified from two bands (Figure $2 \mathrm{~d}^{*}$ ). High-molecular-weight bands likely represent oligomeric forms of TCTP, as reported previously, ${ }^{19,20}$ as the addition of a strong reducing agent (TCEP) to SSC-Apo before protein separation decreased the intensity of high-molecular-weight forms and increased the intensity of the 23-kDa band (Figure 3c, middle and left panels). Also, electrophoresis of recombinant TCTP demonstrated the presence of high-molecular-weight bands (Figure 3c, right panel), indicating dimerization. Of note, recombinant TCTP contains a GST tag, therefore, explaining the higher molecular weights of monomeric and oligomeric forms. Known sequences of importance in TCTP oligomerization ${ }^{19}$ were present within recombinant TCTP. Collectively, these results confirmed that caspase(s) activation in EC enhances the secretion of the nanovesicle marker TCTP.

To confirm the increased release of nanovesicles by apoptotic EC, ultrastructural studies were performed on nanovesicle fractions isolated from SSC-Apo (Figure 3d). Immunogold labeling revealed the presence of TCTP 
Table 1 List of proteins identified exclusively in SSC-Apo (compared with SSC-No-Apo) by two distinct LC-MS/MS approaches

\begin{tabular}{|c|c|c|c|c|c|c|c|}
\hline \multirow[b]{2}{*}{ No. } & \multirow[b]{2}{*}{$\begin{array}{l}\text { Proteins identified by two } \\
\text { LC-MS/MS approaches }\end{array}$} & \multirow[b]{2}{*}{$\begin{array}{l}\text { Gene } \\
\text { name }\end{array}$} & \multicolumn{3}{|c|}{$\begin{array}{l}\text { Type of LC-MS/MS } \\
\text { approach }\end{array}$} & \multirow[b]{2}{*}{$\begin{array}{l}\text { Secretion signal } \\
\text { sequence }\end{array}$} & \multirow[b]{2}{*}{$\begin{array}{l}\text { Mode of } \\
\text { secretion }\end{array}$} \\
\hline & & & $\begin{array}{l}\text { Functional } \\
\text { antiapoptotic fraction }\end{array}$ & 2D-LC & SDS-PAGE & & \\
\hline 1 & TCTP & tpt1 & $x$ & & $x$ & NO & Exosome $^{16,18}$ \\
\hline 2 & $\begin{array}{l}\text { a-SNAP N-ethylmaleimide-sensitive factor } \\
\text { attachment protein alpha NSF }\end{array}$ & Napa & $x$ & & $\mathrm{x}$ & NO & Exosome $^{32}$ \\
\hline 3 & Insulin-like growth factor II receptor & igf2r & $x$ & $x$ & & YES & Exosome $^{32}$ \\
\hline 4 & Aldehyde dehydrogenase 1 & aldh1a1 & & $\mathrm{x}$ & $\mathrm{x}$ & NO & Exosome $^{32}$ \\
\hline 5 & Heat shock $70 \mathrm{kDa}$ protein 1 -like & hspa1l & & $\mathrm{x}$ & $\mathrm{x}$ & NO & Exosome $^{21}$ \\
\hline 6 & Epidermal growth factor & Egf & & $x$ & $x$ & YES & Shedding, \\
\hline 7 & L1 cell adhesion molecule & $11 \mathrm{cam}$ & & $\mathrm{x}$ & $\mathrm{x}$ & YES & Exosome ${ }^{33}$ \\
\hline 8 & $\begin{array}{l}\text { Low density lipoprotein receptor-related } \\
\text { protein } 2\end{array}$ & Irp2 & & $\hat{x}$ & $\hat{x}$ & YES & Exosome $^{32}$ \\
\hline 9 & MHC class II DQ & hla-dqa1 & & $x$ & $\mathrm{x}$ & YES & Exosome $^{34}$ \\
\hline 10 & $\begin{array}{l}\text { Transforming growth factor, } \\
\beta \text { receptor III (betaglycan, } 300 \mathrm{kDa} \text { ) }\end{array}$ & $\operatorname{tgfbr3}$ & & $x$ & $x$ & YES & Exosome $^{32}$ \\
\hline 11 & Ash1 (absent, small or homeotic)-like & ash1I & & $x$ & $x$ & NO & ER microsome $e^{35}$ \\
\hline 12 & $\begin{array}{l}\text { ATPase } \mathrm{H}+/ \mathrm{K}+\text { exchanging alpha } \\
\text { polypeptide }\end{array}$ & Atp4a & & $x$ & $\mathrm{x}$ & NO & $\begin{array}{l}\text { Tubulovesicules } \\
\text { and apical } \\
\text { membrane }\end{array}$ \\
\hline 13 & ADAMTS4 & Adamts4 & & $x$ & $x$ & YES & $\begin{array}{l}\text { Trans Golgi } \\
\text { network }^{37}\end{array}$ \\
\hline 14 & Tissue type plasminogen activator & Plat & & $\mathrm{x}$ & $x$ & YES & $\begin{array}{l}\text { Small dense } \\
\text { vesicles }^{38}\end{array}$ \\
\hline 15 & $\begin{array}{l}\text { Eukaryotic translation initiation } \\
\text { factor } 4 \gamma\end{array}$ & Eif4g1 & $x$ & & $x$ & NO & Undescribed \\
\hline 16 & $\begin{array}{l}\text { Golgi autoantigen, golgin subfamily a, } \\
\text { member } 2\end{array}$ & golga2 & $x$ & $x$ & & NO & Undescribed \\
\hline 17 & Myotrophin & Mtpn & $\mathrm{x}$ & $\mathrm{x}$ & & NO & Undescribed \\
\hline 18 & $\begin{array}{l}\text { Anillin, actin binding protein } \\
\text { (scraps homolog, Drosophila) }\end{array}$ & Anln & & $x$ & $\mathrm{x}$ & NO & Undescribed \\
\hline 19 & $\begin{array}{l}\text { ATP-binding cassette, sub-family C } \\
\text { (CFTR/MRP), member } 2\end{array}$ & $a b c c 2$ & & $x$ & $x$ & NO & Undescribed \\
\hline 20 & Breast cancer 2 , early onset & brca2 & & $x$ & $x$ & NO & Undescribed \\
\hline 21 & Coenzyme Q4 homolog & $\operatorname{cog} 4$ & & $\mathrm{x}$ & $\mathrm{x}$ & NO & Undescribed \\
\hline 22 & Heart muscle iNOS & NOs2a & & $\mathrm{x}$ & $\mathrm{x}$ & NO & Undescribed \\
\hline 23 & Spindlin-like protein 2 & spin2a & & $\mathrm{x}$ & $\mathrm{x}$ & NO & Undescribed \\
\hline 24 & Very large inducible GTPase 1 & gvin1 & & $\mathrm{x}$ & $\mathrm{x}$ & NO & Undescribed \\
\hline 25 & Integrin $\alpha 10$ subunit & itga10 & & $\mathrm{x}$ & $\mathrm{x}$ & YES & Undescribed \\
\hline 26 & $\begin{array}{l}\text { Low density lipoprotein-receptor } \\
\text { related protein } 1\end{array}$ & Irp1 & & $x$ & $x$ & YES & Undescribed \\
\hline 27 & Phospholipase A2, group IID & Pla2g2d & & $\mathrm{x}$ & $\mathrm{x}$ & YES & Undescribed \\
\hline
\end{tabular}

Abbreviations: iNOS, inducible nitric oxide synthase; TCTP; translationally controlled tumor protein. Protein and gene names were obtained from http://ca.expasy.org/ sprot/. Information on secretion signal sequence was obtained from SPdb, a signal peptide database on the following website: http://proline.bic.nus.edu.sg/spdb/. The mode of secretion for each protein was obtained from articles indexed in PubMed. Undescribed denotes proteins for which information concerning their mode of secretion was not described in the literature

on nanovesicles of $30-100 \mathrm{~nm}$ released by apoptotic EC (Figure 3d) and within multivesicular bodies (MVB) in apoptotic EC (Supplementary Figure S1a). Morphometric quantification of MVB-like structures in apoptotic and nonapoptotic EC demonstrated a 7-fold increase in the volume density of MVB-like structures within apoptotic EC (Supplementary Figure S1b). These data suggested that TCTPbearing nanovesicles produced by apoptotic EC originated, at least in part, from MVB, consistent with the classical pathways of exosomal nanovesicle formation.

Exosome-like nanovesicles released by apoptotic EC have an antiapoptotic function. We then evaluated whether exosome-like nanovesicles participate in the antiapoptotic activity of the endothelial apoptotic secretome. Equal volumes of SSC-Apo and SSC-No-Apo were ultracentrifuged, supernatants were collected and exosomal pellets were resuspended in the initial volume of serum-free medium. Total unfractionated SSC-Apo and the corresponding supernatant and exosomal fraction significantly inhibited VSMC apoptosis compared with an equal volume of SSC-No-Apo (Figure 3e and Supplementary Figure S1c).

We also considered the possibility that exosome-like nanovesicles produced by apoptotic EC represent unusually small apoptotic blebs. In that event, the functional activity of apoptotic blebs and exosome-like nanovesicles should be similar. Apoptotic blebs were purified by centrifugation from SSC-Apo, and depletion was confirmed by flow cytometry (Figure 2b). VSMC exposed to unfractionated SSC-Apo (Total) or to nanovesicle fractions resuspended in the initial volume of serum-free medium mounted a significant antiapoptotic response as compared with SSC-No-Apo (Figure 3e). However, VSMC exposed to apoptotic blebs resuspended in the initial volume of serum-free medium failed 
to develop an antiapoptotic response, whereas VSMC exposed to SSC-Apo depleted of apoptotic blebs did so (Figure 3f). Also, TCTP and TSG 101 levels were undetectable in apoptotic blebs isolated from SSC-Apo, whereas both markers were enriched in the nanovesicle fraction (Figure $3 \mathrm{~g}$ ). These results confirmed that apoptotic blebs and exosomelike nanovesicles produced by apoptotic EC represent two distinct entities, with different protein composition and function.

TCTP is a novel antiapoptotic component of the apoptotic secretome. We then sought to identify specific protein(s) involved in the antiapoptotic activity of exosomelike nanovesicles released by apoptotic EC. Three proteins known to be secreted through nanovesicles were identified concomitantly through functional (antiapoptotic) and comparative proteomics strategies (Table 1). Among these, TCTP appeared to be the most likely antiapoptotic candidate as it has known antiapoptotic activity in a number of cell types. Small interfering RNAs (siRNAs) were used to silence TCTP expression in EC, with the aim of reducing the release of TCTP-bearing nanovesicles by apoptotic EC. TCTP silencing reduced intracellular TCTP levels and inhibited TCTP release in nanovesicle extracts (Figure $4 \mathrm{a}$ and Supplementary Figure S3) but did not modulate the apoptosis of serum-starved EC (Figure 4b). TCTP-silenced EC or control EC were employed to condition equal volumes of serum-free media. Unfractionated SSC-Apo generated by

Table 2 List of all other exosomal proteins identified in SSC-Apo and SSC-No-Apo by one proteomic approach

\begin{tabular}{|c|c|c|c|c|c|c|}
\hline \multirow[b]{2}{*}{ No. } & \multirow[b]{2}{*}{$\begin{array}{l}\text { Proteins identified by one LC-MS/MS } \\
\text { approach }\end{array}$} & \multirow[b]{2}{*}{ Gene name } & \multicolumn{3}{|c|}{ Type of LC-MS/MS approach } & \multirow[b]{2}{*}{ References } \\
\hline & & & $\begin{array}{l}\text { Functional } \\
\text { antiapoptotic Fraction }\end{array}$ & 2D-LC & SDS-PAGE & \\
\hline \multicolumn{7}{|c|}{ Exosomal proteins identified exclusively in SSC-Apo } \\
\hline 1 & Decay-accelerating factor & Cd55 & $\mathrm{x}$ & & & 34,39 \\
\hline 2 & Alix & Pdcd6ip & & & $x$ & 34,39 \\
\hline$\overline{3}$ & Milk fat globule-EGF factor8/lactadherin & Mfge8 & & & $\mathrm{x}$ & 34,39 \\
\hline 4 & Integrin $\beta 1$ & Itgb1 & & $\mathrm{x}$ & & 34,39 \\
\hline 5 & Integrin $\alpha 3$ & Itga3 & & $\mathrm{x}$ & & 34,39 \\
\hline 6 & Heat shock protein HSP $90-\alpha$ & Hsp90aa1 & & & $x$ & 34,39 \\
\hline 7 & Flotillin 1 & Flot1 & & & $x$ & 34,39 \\
\hline 8 & Lysosomal-associated membrane protein 3 & Cd63 & & & $\mathrm{x}$ & 34,39 \\
\hline 9 & Actinin & Actn1 & & & $\mathrm{x}$ & 32,40 \\
\hline 10 & ATP citrate lyase & Acly & & & $\mathrm{x}$ & 32,40 \\
\hline 11 & Ezrin & Vil2 & & & $\mathrm{x}$ & 32,40 \\
\hline 12 & Elongation factor $1-\alpha 1$ & Eef1a1 & & & $x$ & 34,39 \\
\hline \multicolumn{7}{|c|}{ Exosomal proteins identified exclusively in SSC-No-Apo } \\
\hline 1 & Integrin $\beta 2$ & Itgb2 & & & $x$ & 32,40 \\
\hline 2 & Rab7 & Rab7 & & & $\mathrm{x}$ & 32,40 \\
\hline
\end{tabular}

A total of 12 exosomal proteins additional to those listed in Table 1 were identified only in SSC-Apo, whereas 2 exosomal proteins were identified only in SSC-No-Apo. Proteins were considered as 'classically exosome associated' when reported as exosomal components in at least two independent reports based on recent literature

\begin{abstract}
Figure 3 Caspase(s) activation in apoptotic EC fosters the release of antiapoptotic exosome-like nanovesicles. (a) Immunoblots for TCTP, TSG 101, Grp96 and tubulin in cytosolic $(25 \mu \mathrm{g})$ and total protein extracts from equal volumes $(1 \mathrm{ml})$ of total serum-free media (Total SSC) conditioned by equal numbers of EC serum starved for $1-4 \mathrm{~h}$. Ponceau red staining is representative of total protein content. Representative of three experiments. (b) Immunoblots for TCTP in proteins precipitated from equal volumes of serum-free media conditioned by equal numbers of apoptotic (Apo) and non-apoptotic EC (No-Apo): 1 ml of unfractionated serum-free conditioned media (Total SSC), exosome-like nanovesicle fraction (Exo) purified from $12 \mathrm{ml}$ of total serum-free conditioned media and nanovesicle-free supernatant (SN) corresponding to the soluble fraction of ultracentrifuged serum-free conditioned media (12 ml). Representative of three experiments. (c) Immunoblots for TCTP. Left panel: Proteins isolated from $1 \mathrm{ml}$ of SSC-Apo compared with $1 \mathrm{ml}$ of SSC-Apo supplemented with TCEP 10\% (SSC-Apo + TCEP) before electrophoresis. TCEP decreased the intensity of high-molecular-weight TCTP and increased the intensity of the non-oligomeric form (monomer $23 \mathrm{kDa}$ ). Middle panel: Quantification by densitometry of TCTP oligomers and monomer from three immunoblots. ${ }^{*} P \leq 0.005$ versus SSC-Apo. Right panel: A total of $0.1 \mu \mathrm{g}$ of GST-tagged recombinant TCTP (37 kDa) showing formation of oligomers. (d) Electron micrographs. Exosome-like nanovesicles purified from SSC-Apo (cleared of cell debris and apoptotic blebs). Left panel: Immunogold labeling of nanovesicle extracts revealed TCTP antigenic sites on the surface of a nanovesicle. Bar: $25 \mathrm{~nm}$. Uranyl oxalate and methyl cellulose-uranyl acetate negative staining. Right panel: Electron micrograph presenting a population of exosome-like nanovesicles positive for TCTP immunogold labeling. Bar: $100 \mathrm{~nm}$. (e) Percentage of apoptotic cells as defined by increased chromatin condensation without cell membrane permeabilization (as evaluated with $\mathrm{HO}$ and $\mathrm{PI}$ staining) in VSMC exposed for $24 \mathrm{~h}$ to $0.5 \mathrm{ml}$ of: $\mathrm{normal}$ medium ( $\mathrm{N}$ ), serum-free medium (SS), unfractionated serum-free media (Total) conditioned either by apoptotic EC (SSC-Apo) or non-apoptotic EC (SSC-No-Apo), as described in Figure 2a, exosome-like fraction (Exo) purified from $12 \mathrm{ml}$ of total serum-free conditioned media and resuspended in $12 \mathrm{ml}$ of RPMI (SS) and the corresponding nanovesicle-free supernatant (SN). ${ }^{*} P \leq 2.0 \times 10^{-5}$ versus SSC-Apo, $n \geq 6$. (f) Percentage of apoptotic cells as defined by increased chromatin condensation without cell membrane permeabilization (as evaluated with $\mathrm{HO}$ and PI staining) in VSMC exposed for $24 \mathrm{~h}$ to $0.5 \mathrm{ml}$ of: normal medium (N), serum starvation (SS), total serum-free medium conditioned by apoptotic EC (total), supernatant (SSC WO blebs) after removal of apoptotic blebs by centrifugation at $50000 \times g(12 \mathrm{ml})$ and apoptotic blebs (SS + blebs) purified from $12 \mathrm{ml}$ of total conditioned medium by centrifugation and resuspended in $12 \mathrm{ml}$ of $\mathrm{SS}$. ${ }^{\star} P \leq 1 \times 10^{-11}$ versus SS, $n=8$. (g) Immunoblots for TCTP and TSG 101 in apoptotic blebs (Blebs) and exosome-like nanovesicle fraction (Exo) isolated from $35 \mathrm{ml}$ of serum-free media conditioned by apoptotic serum-starved EC. The immunoblot for TCTP corresponds to two parts of the same gel. Representative of three experiments. The color reproduction of this figure is available on the html full text version of the manuscript
\end{abstract}


a SS tx :

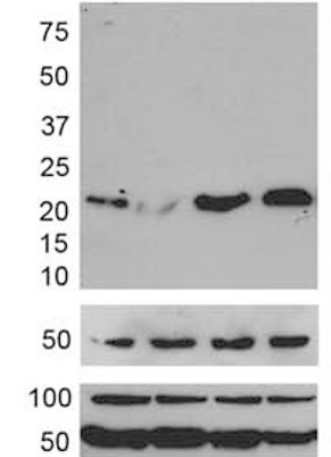

Total SSC

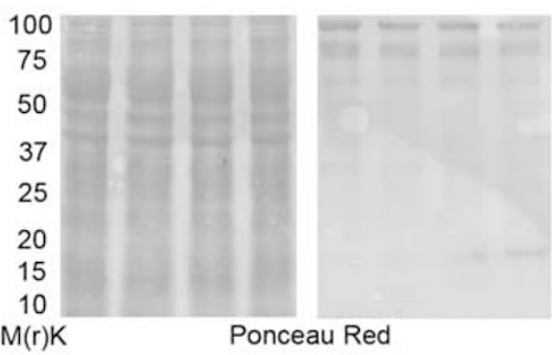

b $\frac{\text { Total SSC }}{\text { SSC: }} \frac{\text { Exo No-Apo }}{\text { Apo No-Apo }} \frac{\text { ANo No-Apo }}{\text { Apo No Non }}$

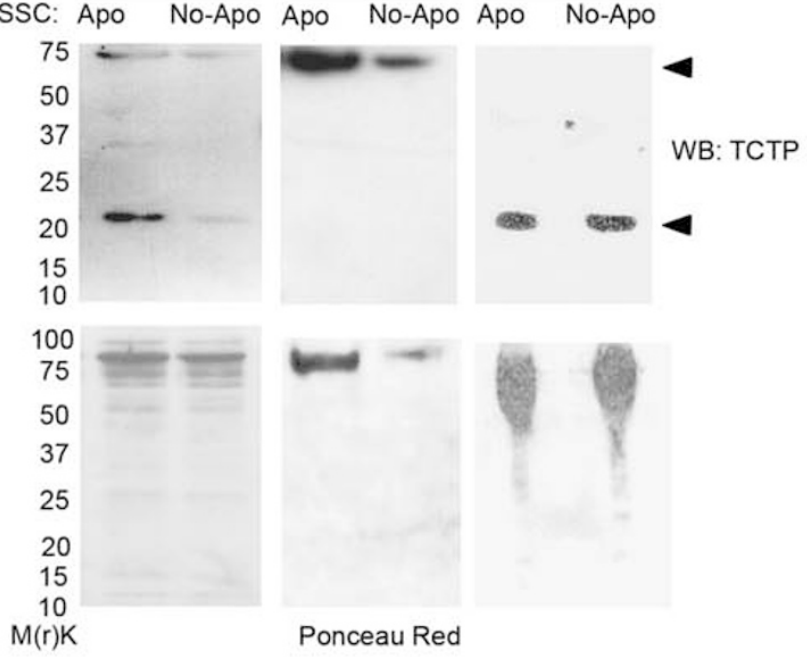

c

SSC SSC

Apo Apo +

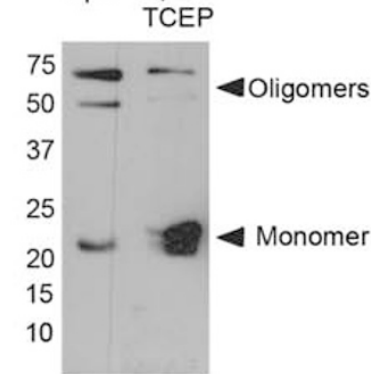

$M(r) K$ WB: TCTP

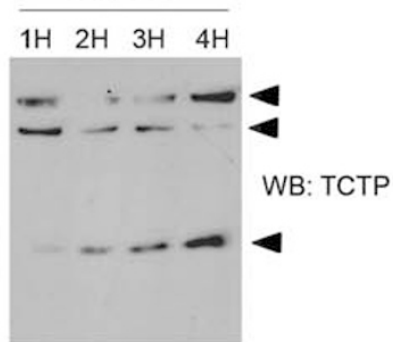

WB: TSG 101

WB: Grp96 WB: Tubulin

WB: Tubulin d
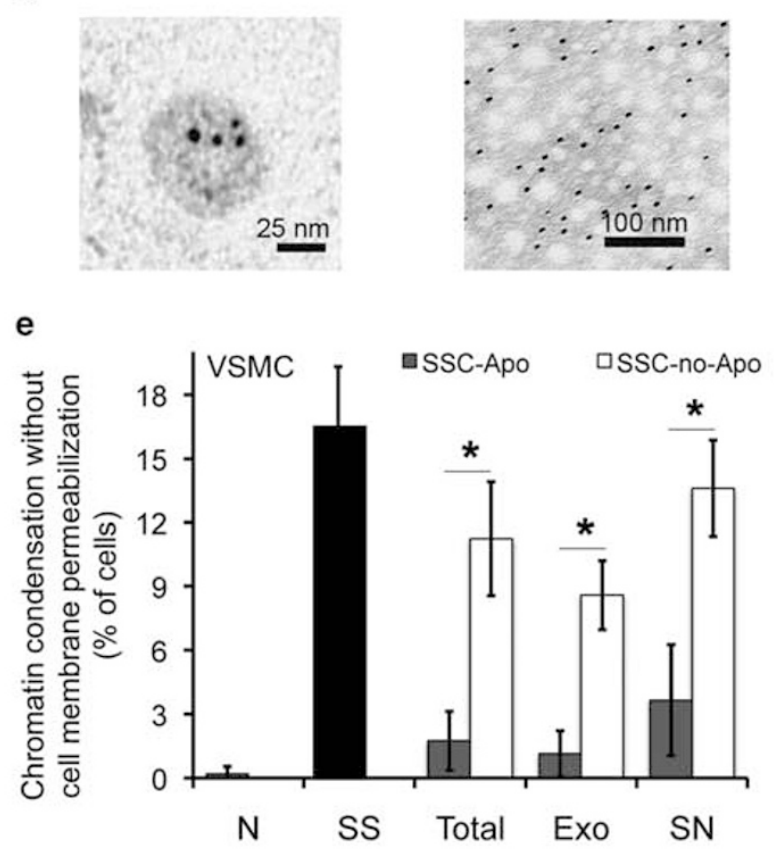

f

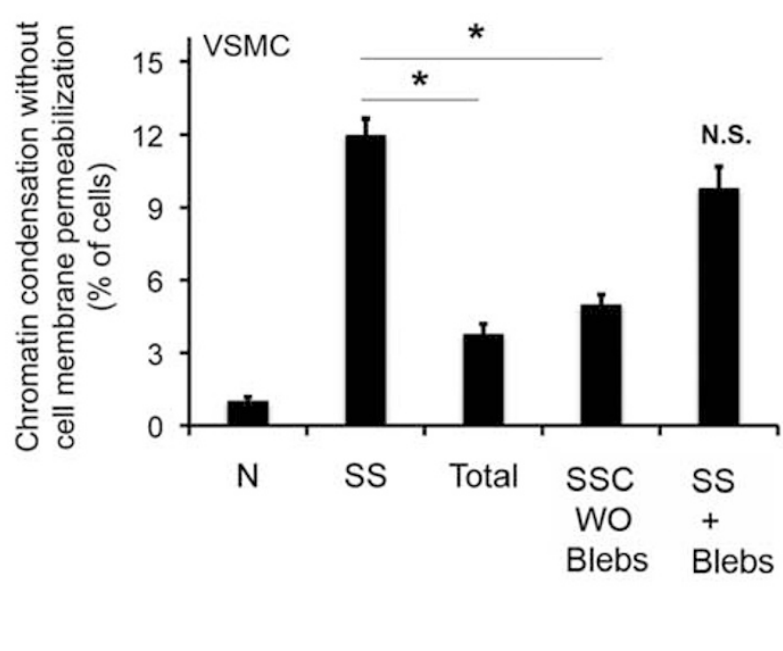

g

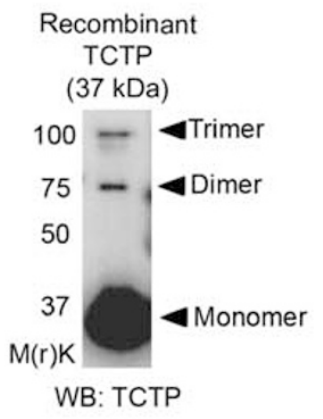

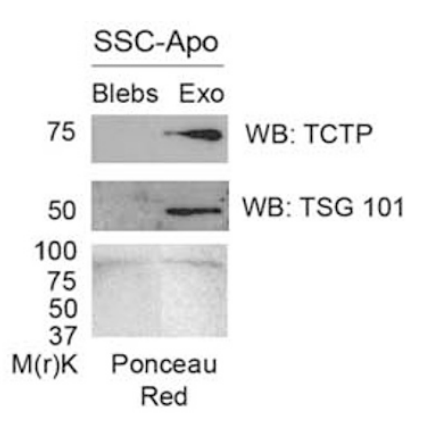




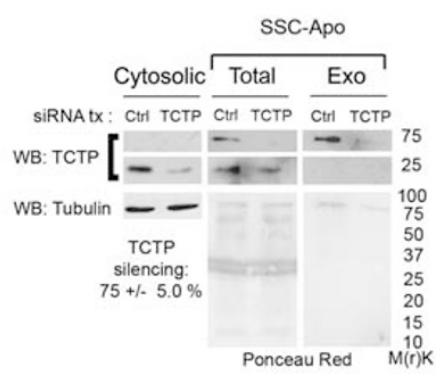

b

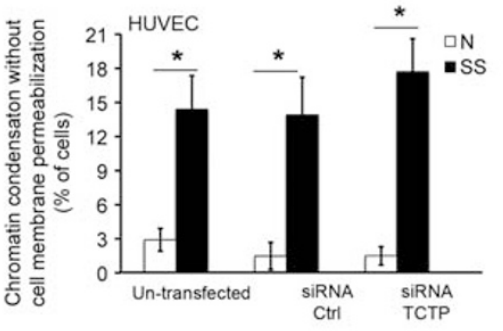

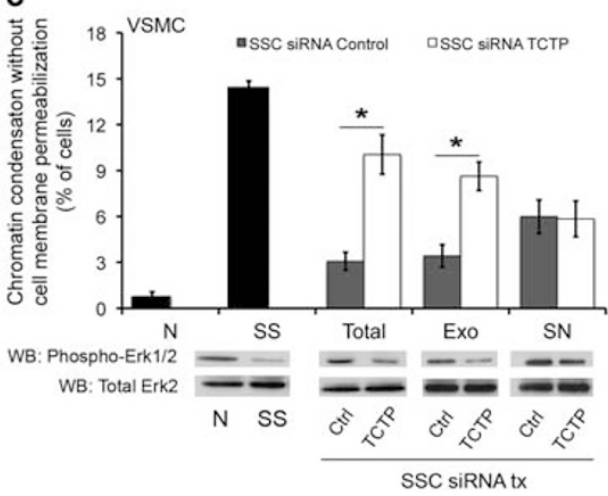

d

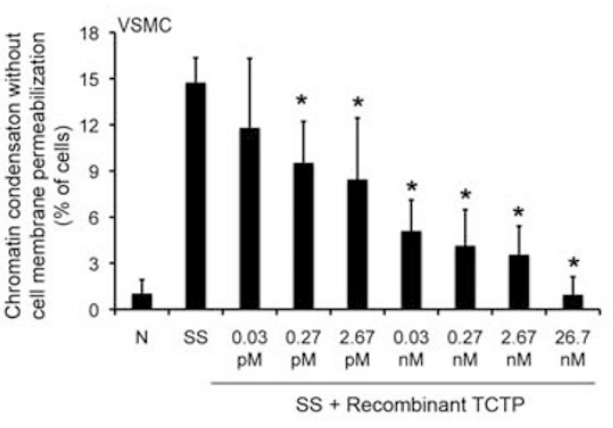

e

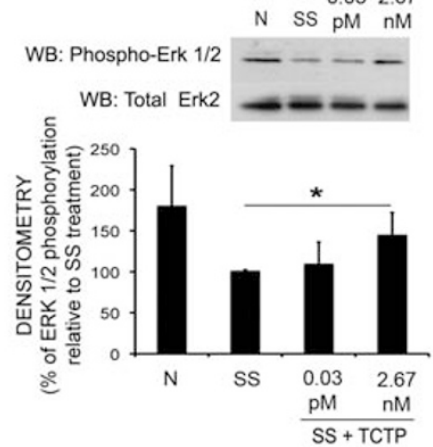

f

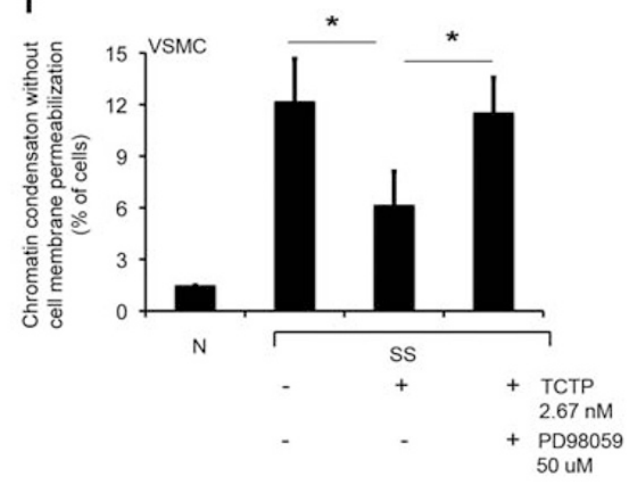

Figure 4 TCTP is a novel antiapoptotic component of the apoptotic secretome. (a) Equal numbers of EC were transfected with control siRNA (siRNA Ctrl) or TCTP siRNA (siRNA TCTP) and serum starved for $4 \mathrm{~h}$ in equal volumes of serum-free media. Immunoblots for TCTP in: $25 \mu \mathrm{g}$ of cytosolic extracts (Cytosolic), proteins precipitated from $1 \mathrm{ml}$ of unfractionated serum-free conditioned media (Total) and exosome-like nanovesicle fraction (Exo) purified from $25 \mathrm{ml}$ of total conditioned media. Tubulin is shown as a loading control for cytosolic extracts. Ponceau red staining indicates total protein content. Representative of three experiments. (b) Percentage of apoptotic cells as defined by increased chromatin condensation without cell membrane permeabilization (as evaluated with $\mathrm{HO}$ and PI staining) in EC pretreated with either control siRNA or TCTP siRNA exposed to normal medium or SS for $4 \mathrm{~h} .{ }^{*} P \leq 0.05$ versus $\mathrm{N}, n=6$. (c) Percentage of apoptotic cells as defined by increased chromatin condensation without cell membrane permeabilization (as evaluated with $\mathrm{HO}$ and PI staining) in VSMC exposed for $24 \mathrm{~h}$ to $0.5 \mathrm{ml}$ of: normal medium $(\mathrm{N})$, serum starvation (SS), total unfractionated serum-free media (Total) conditioned by apoptotic EC transfected with either control siRNA (SSC siRNA Ctrl) or TCTP siRNA (SSC siRNA TCTP), exosome-like nanovesicle fraction (Exo) purified from $12 \mathrm{ml}$ of total unfractionated serum-free conditioned media and resuspended in $12 \mathrm{ml}$ of RPMI (SS) or the corresponding nanovesicle-free supernatant (SN). ${ }^{\star} P<1 \times 10^{-5}$ versus siRNA control, $n \geq 7$. Lower panel: Immunoblots for phosphorylated and total ERK $1 / 2$ in cytosolic extracts ( $\left.25 \mu \mathrm{g}\right)$ of VSMC exposed for 30 min to normal medium (N), serum starvation (SS), Total, Exo and SN from SSC siRNA control and SSC siRNA TCTP. Immunoblots are representative of three experiments. (d) Percentage of apoptotic cells as defined by increased chromatin condensation without cell membrane permeabilization (as evaluated with $\mathrm{HO}$ and PI staining) in VSMC exposed for $24 \mathrm{~h}$ to: normal medium (N), serum starvation in RPMI (SS) and recombinant TCTP (0.03 pM to $26.7 \mathrm{nM}$ ) resuspended in RPMI (SS). ${ }^{*} P \leq 0.009$ versus SS, $n \geq 4$. (e) Upper panel: Immunoblots for phosphorylated and total ERK $1 / 2$ in cytosolic extracts $(25 \mu \mathrm{g})$ of VSMC exposed for 30 min to $0.03 \mathrm{pM}$ and $2.67 \mathrm{nM}$ of recombinant TCTP resuspended in RPMI (SS). Representative of three experiments. Lower panel: Quantification by densitometry for the percentage of ERK 1/2 phosphorylation relative to SS treatment in VSMC. ${ }^{*} P \leq 0.02$ versus SS, $n=3$. (f) Percentage of apoptotic cells as defined by increased chromatin condensation without cell membrane permeabilization (as evaluated with $\mathrm{HO}$ and PI staining) in VSMC exposed for $24 \mathrm{~h}$ to: normal medium (N), serum starvation in RPMI (SS), recombinant TCTP $26.7 \mathrm{nM}$ resuspended in RPMI (SS) and vehicle (DMSO) or recombinant TCTP $26.7 \mathrm{nM}$ resuspended in RPMI (SS) + PD98059 $50 \mu \mathrm{M} .{ }^{*} P \leq 0.0008$ versus SS or PD 98059; $n \geq 8$. The color reproduction of this figure is available on the html full text version of the manuscript

TCTP-silenced EC (SSC siRNA TCTP) or its corresponding nanovesicle fraction failed to inhibit VSMC apoptosis, whereas control SSC-Apo (SSC siRNA Ctrl) or its corresponding nanovesicle fraction did so (Figure 4c). There was, however, no difference in the antiapoptotic activity of nanovesicle-free supernatants conditioned by serumstarved, TCTP-silenced and control EC (Figure 4c).

As ERK $1 / 2$ activation is a characteristic feature of the antiapoptotic phenotype of neointimal cells, we evaluated whether TCTP induced ERK 1/2-dependent antiapoptotic pathways in VSMC. VSMC exposed to medium conditioned by siRNA TCTP (unfractionated and exosomal fraction) showed reduced ERK 1/2 phosphorylation in comparison with their respective controls (SSC siRNA Ctrl) (Figure 4c, lower panel). ERK $1 / 2$ phosphorylation was similar in VSMC exposed to supernatants derived from media conditioned by TCTP-silenced or control EC. In addition, serum-starved VSMC exposed to recombinant TCTP (which included peptides identified by in-gel LC-MS/MS, Supplementary Figure S1d) manifested dose-dependent inhibition of apoptosis (Figure 4d) in association with increased ERK 1/2 activation (Figure 4e). The concentration of TCTP released by apoptotic EC in SSC-Apo was within the bioactive range (Supplementary Figure S1e). Inhibition of ERK 1/2 activation in VSMC with PD98059 blocked the antiapoptotic activity of recombinant TCTP (Figure 4f). Collectively, these results 
a

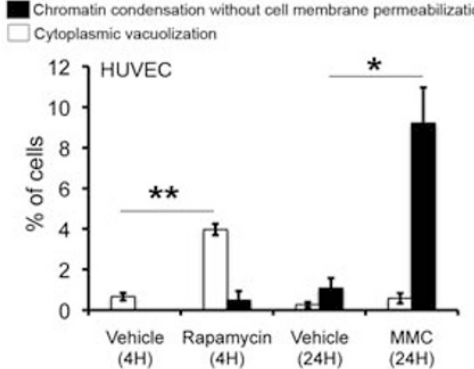

b
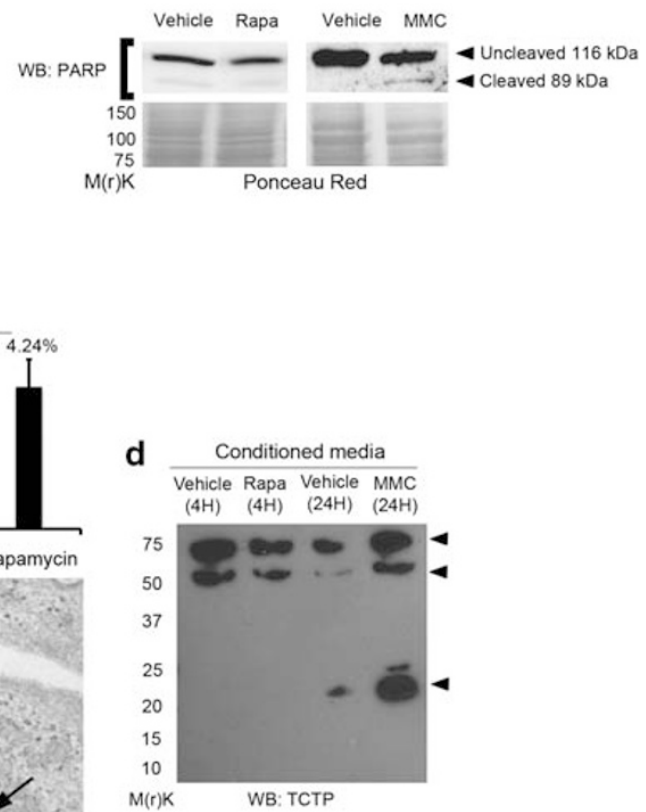

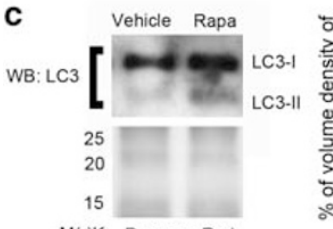

$M(r) K$ Ponceau Red

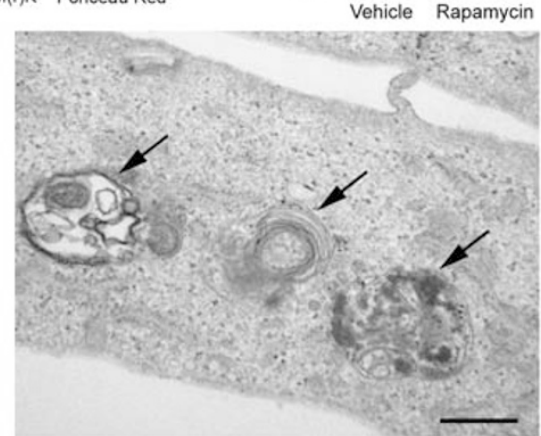

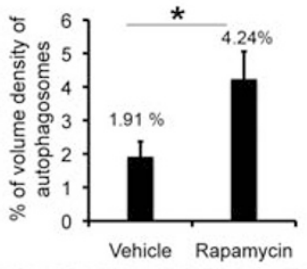

Figure 5 TCTP export is specific of apoptotic cell death. (a) Quantification of apoptotic and autophagic cells by fluorescence microscopy in HO-PI and acridine orangestained EC exposed to: MMC $0.01 \mathrm{mg} / \mathrm{ml}$ in normal medium or vehicle in normal medium for $24 \mathrm{~h}$ and rapamycin $0.1 \mu \mathrm{g} / \mathrm{ml}$ in normal medium or vehicle in normal medium for $4 \mathrm{~h}$. Apoptosis refers to the percentage of cells with increased chromatin condensation without cell membrane permeabilization (as evaluated with $\mathrm{HO}$ and $\mathrm{PI}$ staining). Autophagy referred to the percentage of cells with increased formation of cytoplasmic vacuolization as assessed by acridine orange staining. ${ }^{*} P=1 \times 10^{-6}$ versus vehicle (24h), $n=3$. ${ }^{\star *} P=1 \times 10^{-6}$ versus vehicle $(4 \mathrm{~h}), n=6$. (b) Immunoblots for PARP in cytosolic extracts of EC treated with rapamycin or MMC as described in (a). (c) Left upper panel: Immunoblots showing conversion of LC3-I to LC3-II in EC treated with rapamycin $0.1 \mu \mathrm{g} / \mathrm{ml}$ or vehicle in normal medium for $4 \mathrm{~h}$. Electron microscopy for morphological characterization of autophagosomes. Right upper panel: Quantification of volume density of autophagosomes in EC treated with rapamycin $0.1 \mu \mathrm{g} / \mathrm{ml}$ or vehicle as described in (a). A total of 25 cytoplasmic fields were evaluated for each condition. ${ }^{*} P=0.017$. Lower panel: Three different autophagosomes in rapamycine-treated EC (arrows) used for quantification. Bar: $0.5 \mu \mathrm{m}$. (d) Immunoblots for TCTP in $1 \mathrm{ml}$ of total serum-free media (cleared of cell debris and apoptotic blebs) conditioned in equal volumes and by equal numbers of EC exposed to MMC and rapamycin, as described in (a). Representative of three experiments. Ponceau red is shown as loading control for $\mathbf{b}$ and $\mathbf{c}$. The color reproduction of this figure is available on the $\mathrm{html}$ full text version of the manuscript

establish the importance of TCTP in the activation of an ERK 1/2-dependent antiapoptotic response in VSMC.

Increased TCTP export is characteristic of apoptotic cell death. To evaluate whether TCTP release by apoptotic cells was specific to EC, VSMC were serum starved in the presence of ZVAD-FMK or vehicle (DMSO). SS induced an apoptotic response in VSMC, which was blocked in the presence of ZVAD-FMK (Supplementary Figure S1f). Elevated TCTP levels were found in medium conditioned by apoptotic VSMC, compared with an equal volume of medium conditioned in the presence of ZVAD-FMK (Supplementary Information Figure S1f, lower panel). These data suggest that caspase activation favors TCTP export in different cell types.

As nutrient deprivation is a potent autophagic stimulus, we also considered the possibility that autophagy could contribute to TCTP export. EC exposed to rapamycin, a specific inhibitor of mammalian target of rapamycin, showed characteristic autophagic features as evaluated by acridine orange staining (Figure 5a), LC3 processing and electron microscopy (Figure $5 \mathrm{c}$ ) in absence of apoptotic features as evaluated with $\mathrm{HO}-\mathrm{PI}$ staining (Figure $5 \mathrm{a}$ ) and PARP cleavage (Figure $5 \mathrm{~b}$ ). EC exposed to rapamycin failed to increase TCTP release (Figure $5 \mathrm{~d}$ ). Treatment with mitomycin C (MMC), another proapoptotic stimulus, increased the percentage of cells with chromatin condensation (Figure $5 a$ ), PARP cleavage (Figure 5b) and TCTP release (Figure 5d and Supplementary Figure S3). These results point to the activation of specific apoptosis-associated caspase(s) as a novel pathway regulating TCTP export.

Activated caspase-3 is a novel regulator of TCTP export. In serum-starved EC, TCTP export is initiated (Figure 6a) concomitantly with caspase-3 activation (Figure 1d). Biochemical pan-caspase inhibition or specific caspase-3 

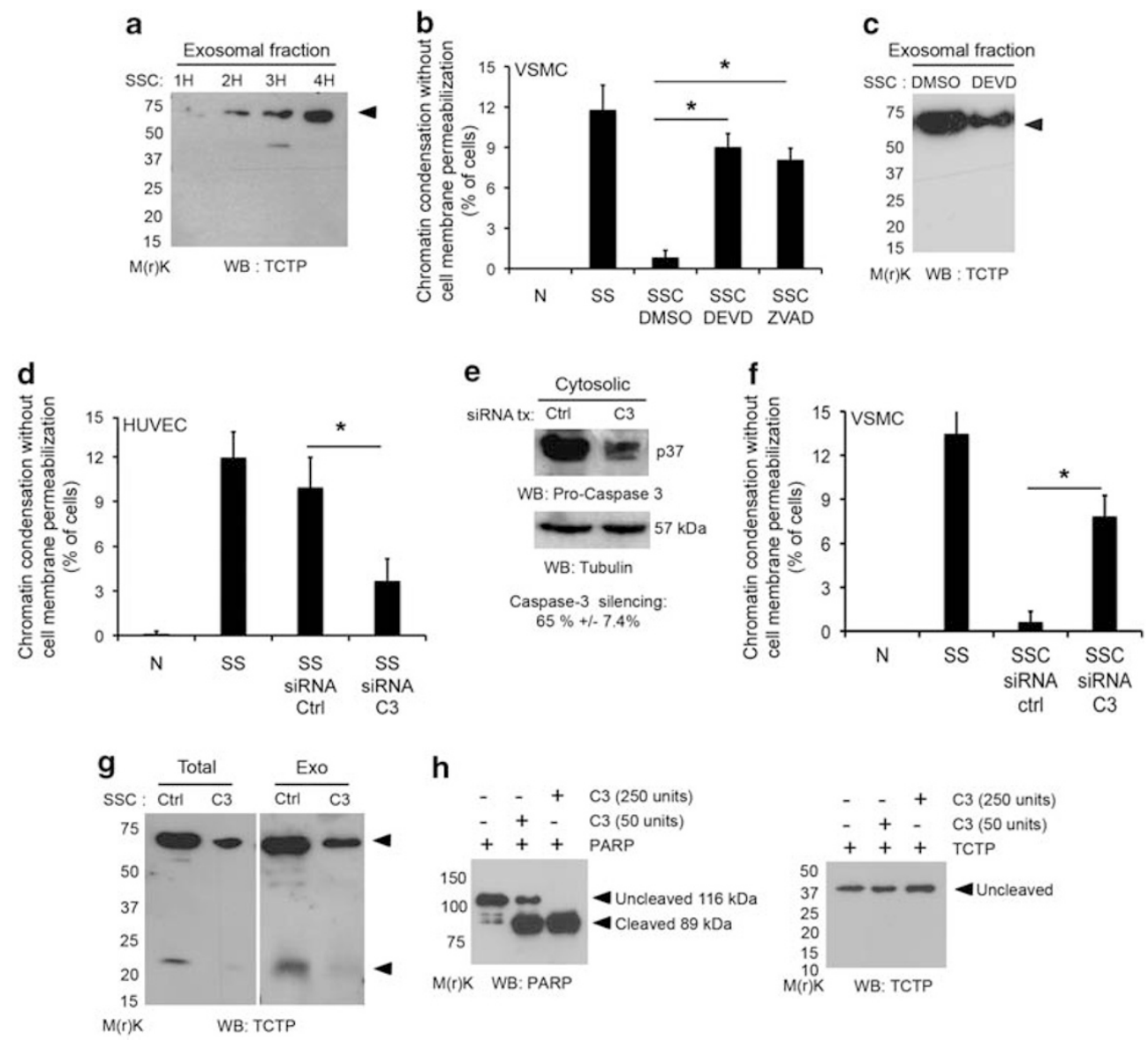

h
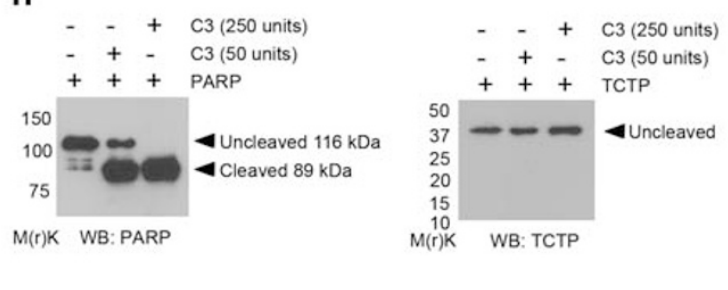

Figure 6 Activated caspase-3 is a novel regulator of TCTP export. (a) Immunoblots for TCTP in exosome-like nanovesicle extracts (Exo) purified from $25 \mathrm{ml}$ of total unfractionated serum-free media conditioned by equal numbers of serum-starved EC in RPMI (SS) for 1-4h. Representative of three experiments. (b) Percentage of apoptotic cells as defined by increased chromatin condensation without cell membrane permeabilization (as evaluated with $\mathrm{HO}$ and PI staining) in VSMC exposed for $24 \mathrm{~h}$ to $0.5 \mathrm{ml}$ of normal medium (N), serum starvation with $0.5 \mathrm{ml}$ of RPMI (SS), total serum-free media conditioned by equal numbers of apoptotic EC (SSC-DMSO) or non-apoptotic EC preincubated with either the caspase-3 inhibitor DEVD-FMK (SSC-DEVD) or the pan-caspase inhibitor ZVAD-FMK (SSC-ZVAD), as described in Figure $1 \mathrm{e}-\mathrm{g}$. ${ }^{*} P<1 \times 10^{-10}$ versus SS, $n \geq 3$. (c) Immunoblots for TCTP in exosome-like nanovesicle extracts (Exo) purified from $12 \mathrm{ml}$ of total unfractionated serum-free media conditioned by equal numbers of EC preincubated as in (b) either with vehicle (SSC-DMSO) or the caspase-3 inhibitor DEVD-FMK (SSC-DEVD) and serum starved for $4 \mathrm{~h}$. Representative of three experiments. (d) Percentage of apoptotic cells as defined by increased chromatin condensation without cell membrane permeabilization (as evaluated with $\mathrm{HO}$ and PI staining) in EC exposed for $4 \mathrm{~h}$ to $0.5 \mathrm{ml}$ of normal medium (N) or serum starvation with RPMI (SS); or EC transfected with either siRNA control (SS siRNA Ctrl) or siRNA caspase-3 (SS siRNA C3) before serum starvation ( $0.5 \mathrm{ml}$ of SS) for $4 \mathrm{~h}$. ${ }^{*} P<0.001$ versus SS siRNA control, $n=3$. (e) Immunoblots for the proform of caspase-3 (p37) in cytosolic extracts $(50 \mu \mathrm{g})$ of EC transfected with caspase-3 siRNA (C3) or control siRNA (Ctrl) and exposed to normal medium for $4 \mathrm{~h}$. Representative of four experiments. Tubulin is shown as loading control. (f) Percentage of apoptotic cells as defined by increased chromatin condensation without cell membrane permeabilization (as evaluated with $\mathrm{HO}$ and $\mathrm{PI}$ staining) in VSMC exposed for $24 \mathrm{~h}$ to: $0.5 \mathrm{ml}$ of normal medium (N), serum starvation with RPMI (SS) and total unfractionated serum-free media conditioned by EC transfected with either siRNA control (SSC siRNA Ctrl) or siRNA C3 (SSC siRNA C3), as described above. ${ }^{*} \leq 0.004$ versus SS, $n \geq 3$. (g) Immunoblots for TCTP in protein extracts precipitated from equal volumes of serum-free media conditioned by equal EC numbers and transfected with siRNA control (Ctrl) or caspase-3 siRNA (C3) and serum starved for $4 \mathrm{~h} ; 1 \mathrm{ml}$ of total unfractionated serum-free media (Total); exosome-like nanovesicle fraction (Exo) purified from $25 \mathrm{ml}$ of conditioned media. Representative of three experiments. (h) Immunoblots for PARP and TCTP exposed to recombinant activated caspase- 3 in vitro. A total of $300 \mathrm{ng}$ (2.6 pmol) of recombinant PARP (left panel) and $270 \mathrm{ng}(7.3 \mathrm{pmol})$ of recombinant TCTP (right panel) were incubated at $37^{\circ} \mathrm{C}$ for $1 \mathrm{~h}$ with 50 and 250 units of activated caspase-3. PARP is a known cleavable substrate of activated caspase- 3 and was used as a positive control. Representative of two experiments

inhibition in serum-starved EC (preincubation with ZVAD-FMK or DEVD-FMK, respectively) concomitantly inhibited EC apoptosis (Figure 1g) and the release of antiapoptotic mediators active on VSMC (Figure 6b). In addition, TCTP levels were reduced in nanovesicle extracts from medium conditioned by serum-starved EC preincubated with DEVDFMK, an irreversible caspase-3 inhibitor, compared with medium conditioned by apoptotic serum-starved EC (Figure 6c and Supplementary Figure S3).

To further address the importance of caspase-3 activation in TCTP export (and potential off-target effects of biochemical caspase inhibitors), siRNAs were used to silence caspase-3 expression in EC. Caspase-3 silencing significantly inhibited the apoptosis of serum-starved EC compared with EC transfected with control siRNAs (Figure $6 \mathrm{~d}$ and e). In turn, VSMC exposed to medium conditioned by caspase-3silenced EC (SSC siRNA C3) failed to mount an antiapoptotic response, whereas VSMC exposed to medium conditioned by control apoptotic EC (SSC siRNA Ctrl) developed an antiapoptotic phenotype (Figure 6f). TCTP levels were lower in unfractionated conditioned medium (Total) and in the nanovesicle fraction (Exo) of medium conditioned by 
caspase-3-silenced EC (SSC siRNA C3) compared with control apoptotic EC (SSC siRNA Ctrl) (Figure $6 \mathrm{~g}$ and Supplementary Figure S3). Finally, we evaluated whether activated caspase-3 could directly cleave TCTP. Recombinant caspase-3 did not show any proteolytic activity toward recombinant TCTP in vitro but did cleave its known substrate PARP (Figure 6h). Also, total and nanovesicle fractions of SSC-Apo failed to show bands lower than the expected $23 \mathrm{kDa}$, ruling out a contribution of TCTP proteolysis. Collectively, these results demonstrate a key role for caspase-3 activation in the release of TCTP by apoptotic EC.

\section{Discussion}

Apoptotic cells actively release a finely regulated set of 'come and eat-me' and 'stay-away' paracrine signals aimed at coordinating the specific recruitment of mononuclear phagocytes. ${ }^{1,2}$ Recent evidence indicates that the paracrine component of the apoptotic program is not limited to the regulation of leukocyte trafficking but also aims at preparing the local microenvironment for remodeling after cell deletion. The present work lends further support to this contention. Taking a multifaceted proteomics approach, we identified a set of 27 proteins released specifically by apoptotic EC (characterized by increased chromatin condensation and exclusion of propidium iodide $(\mathrm{PI})$, loss of mitochondrial integrity and evidence of activated caspase-9 and -3) downstream of caspase activation. Several lines of evidence demonstrated that these proteins are not released as a consequence of cell membrane permeabilization. First, plasma membrane rupture, as evidenced by PI staining, is absent in serum-starved EC, and caspase inhibition does not impact cell membrane permeability. Second, LDH, a classical marker of cell membrane permeabilization, is not increased in medium conditioned by serum-starved apoptotic EC. Finally, the release of proteins by serum-starved EC is a targeted response, as Grp96 and tubulin are not secreted throughout SS, whereas TSG 101 and TCTP are released concomitantly with caspase activation.

Non-classical protein secretion pathways are, at least in part, responsible for the paracrine component of the apoptotic program in EC. Among the 27 proteins identified specifically in medium conditioned by apoptotic EC, more than half did not contain a secretion signal sequence. Also, irrespective of the presence of a secretion signal, more than a third corresponded to typical nanovesicle components. In recent years, the characterization of different types of microparticles and nanovesicles released by live cells has been under intense investigation. Unfortunately, there is yet no widely-accepted classification of the various categories of micro- and nanovesicles. Size, appearance under electron microscopy and intracellular origin are currently key features that differentiate various types of membrane vesicles. Exosomes are small, cup-shaped vesicles of $50-100 \mathrm{~nm}$ diameter. ${ }^{15,21-23}$ Their precursors are intracellular vesicles packaged into MVB, which fuse with the plasma membrane, releasing exosomal vesicles into the extracellular milieu. ${ }^{15,21-23}$ Exosome-like nanovesicles are somewhat smaller $(20-50 \mathrm{~nm})$, of irregular shape and probably stem also from endosomal compartments. ${ }^{15}$ Apoptotic blebs are bigger $(50-500 \mathrm{~nm})$, of hetero- genous shapes and result from budding of the cell membrane. ${ }^{15,24}$ The nanovesicles released by apoptotic EC share many characteristics with exosome-like nanovesicles and exosomes. Immunoblotting confirmed elevated levels of the exosomal markers, TCTP and TSG 101 in medium conditioned by apoptotic EC. Irregular-shaped nanovesicles of sizes within the range described for exosome-like nanovesicles were demonstrated by electron microscopy in medium conditioned by apoptotic EC. Immunogold labeling confirmed that TCTP was present at the surface of these nanovesicles and within MVB of apoptotic EC.

We, nonetheless, considered the possibility that nanovesicles released by apoptotic EC could represent small apoptotic blebs. Several lines of evidence suggest that TCTP-expressing nanovesicles differ from apoptotic blebs. First, TCTP and TSG 101 levels are high in nanovesicle fractions but undetectable in apoptotic bleb extracts. Second, histones, previously described as a specific component of apoptotic blebs ${ }^{15,21}$ were not recovered with any of our proteomics strategies (data not shown), further confirming optimal depletion of apoptotic blebs before MS/MS analysis. Finally, exosome-like nanovesicles purified from medium conditioned by apoptotic EC trigger an antiapoptotic phenotype in VSMC, whereas apoptotic blebs purified from the same medium do not. These results indicate that over the course of apoptosis at least two functionally different types of vesicles are released: apoptotic blebs and nanovesicles. Whether the latter correspond to the enhanced production of exosome-like nanovesicles normally released by live EC or to a different nanovesicle type specific to the apoptotic program, sharing a common intracellular origin and at least some markers with exosome and exosome-like nanovesicles, will be the scope of future investigation.

Our work identifies activated caspase-3 as a novel regulator of TCTP export through the nanovesicle pathway. p53 is known to regulate TSAP- 6 activity, which, in turn, controls exosome production and TCTP release.$^{16-18}$ Here, increased release of nanovesicle constituents was identified by an unbiased proteomics strategy, where media conditioned by caspase-activated or -inactivated EC were compared. Caspase(s) inhibition with ZVAD-FMK impacts the execution phase of apoptosis but does not alter molecular events associated with apoptosis initiation, which includes similar intracellular p53 levels and mitochondrial permeabilization. Hence, p53 cannot be inferred as a regulator of TCTP export in our system. Caspase-3 biochemical inhibition or genetic silencing similarly prevents TCTP export, pointing to a pivotal role for caspase-3 in the release of TCTP-bearing nanovesicles by apoptotic cells.

Several lines of evidence indicate that TCTP plays a functional role in the antiapoptotic activity of nanovesicles released by apoptotic EC. VSMC exposed to TCTP-expressing nanovesicles show increased resistance to apoptosis and activation of ERK 1/2, therefore, recapitulating $a$ neointimal phenotype. ${ }^{3,9}$ TCTP silencing in EC blocks TCTP release and largely decreases both ERK 1/2 activation and resistance to apoptosis in VSMC exposed to nanovesicles produced by apoptotic EC. Finally, recombinant TCTP increases ERK 1/2 activation and inhibits VSMC apoptosis. TCTP is an evolutionarily conserved protein of crucial 
importance during development ${ }^{25}$ and for intracellular apoptosis inhibition. ${ }^{26,27}$ The present work demonstrates that TCTP also displays significant extracellular antiapoptotic activity. These results are in keeping with recent investigations indicating that extracellular TCTP activates ERK 1/2 in basophils.

The present study highlights novel mechanisms of cross talk between dying cells and the local microenvironment. Apoptotic cells actively release apoptotic blebs and exosomelike nanovesicles. The latter, through an antiapoptotic activity on cells of importance in tissue and vascular repair, likely favor remodeling at sites of apoptotic cell deletion. However, a chronic increase in apoptosis could foster sustained activation of the paracrine program, resulting in maladaptive remodeling.

\section{Materials and Methods \\ Cell culture and generation of conditioned media. HUVEC (Clonetics, San Diego, CA, USA) were grown in microvascular EC medium (Clonetics) and used at passages 2-5. WI-38 human fibroblasts (ATCC, Rockville, MD, USA) were grown in FBM (Cambrex, Walkersville, MD, USA) with $10 \%$ inactivated FBS (Medicorp, Montréal, Qc, Canada) and employed at passages 2- 17. A7R5 cells (VSMC), a clonal rat smooth muscle cell line (ATCC), were cultured in DMEM/F12 with $10 \%$ FBS. Serum-free media, conditioned by apoptotic or caspase- inhibited EC, were obtained as described previously. ${ }^{3-8}$ Equal EC numbers $\left(2.5 \times 10^{4}\right.$ cells $/ \mathrm{cm}^{2}$ ) were preincubated for $2 \mathrm{~h}$ in normal medium containing either DMSO (vehicle) or ZVAD-FMK $(100 \mu \mathrm{M})$ or DEVD-FMK $(100 \mu \mathrm{M})$ (R\&D Systems, Minneapolis, MN, USA) for caspase(s) inhibition. Then, the culture medium was changed for serum-free medium RPMI (Gibco, Burlington, Ontario, Canada) and EC were serum starved for $4 \mathrm{~h}$.}

\section{Characterization of the secretome produced by apoptotic EC.} We adopted two comparative proteomics approaches to identify the secretome released by apoptotic EC: 2D-LC-MS/MS and SDS-PAGE LC-MS/MS. ${ }^{14}$ Serumfree media $\left(0.16 \mathrm{ml} / \mathrm{cm}^{2}\right)$ conditioned by apoptotic (SSC-Apo) and non-apoptotic EC preincubated with ZVAD-FMK (SSC-No-Apo) were centrifuged sequentially at $1200 \times g$ and $50000 \times g$ to eliminate cell debris and apoptotic blebs, and depletion was confirmed by flow cytometry (FACScan equipped with CellFit software, Becton-Dickinson, San José, CA, USA) in FL1 and FL2 channels. We also performed a functional analysis of SSC-Apo. A total of $750 \mathrm{ml}$ of medium conditioned by apoptotic EC was fractionated by FPLC, and each fraction was tested for antiapoptotic activity on VSMC. ${ }^{3}$ The antiapoptotic fraction was resolved by SDS-PAGE, and the bands were sequenced with LC-MS/MS.

For 2D-LC-MS/MS analysis, $40 \mathrm{ml}$ of conditioned media cleared of cell debris and apoptotic blebs were concentrated ( $0.5 \mathrm{mg}$ of proteins) and solubilized in $100 \mu \mathrm{l}$ of $40 \mathrm{mM}$ Tris- $\mathrm{HCl}, \mathrm{pH} 8.0,7.5 \mathrm{M}$ urea, $2.0 \mathrm{M}$ thiourea, reduced in the presence of $6 \mathrm{mM}$ DTT, alkylated with $15 \mathrm{mM}$ iodoacetamide and digested overnight with trypsin (Promega, Madison, WI, USA, sequencing grade, estimated trypsin/protein ratio $1: 100)$. The tryptic peptides were resuspended in $55 \%$ acetonitrile, $25 \%$ dimethylformamide and labeled with pentafluorophenyl-4-anilino-4-oxobutanoatedo or pentafluorophenyl-4-anilino-4-oxobutanoate-d5. Labeled samples were combined, desalted, evaporated to dryness, resuspended in $10 \%$ acetonitrile, containing $10 \mathrm{mM}$ ammonium acetate, $\mathrm{pH} 7.0$, and analyzed by weak anion exchange chromatography in a PolyWAX column $(100 \times 1 \mathrm{~mm}, 5 \mu \mathrm{m}, 300 \AA$; PolyLC, Columbia, MO, USA) connected to an Agilent 1100 Series LC system (Santa Clara, CA, USA). Peptides were eluted by a gradient of 0-1 M NaCl in $10 \mathrm{mM}$ ammonium acetate, $\mathrm{pH} 6.0$, containing $10 \%$ acetonitrile, at a flow rate of $20 \mu \mathrm{l} / \mathrm{min}$. A total of 36 fractions of $80 \mu \mathrm{l}$ were collected and acidified with formic acid. A total of $30 \mu$ from each fraction were analyzed by LC-MS/MS.

SDS-PAGE LC-MS/MS analysis was performed essentially as outlined elsewhere ${ }^{14}$. Proteins $(125 \mu \mathrm{g})$ were solubilized in $4 \times$ Laemmli buffer $(62.5 \mathrm{mM}$ Tris-HCl, pH 6.8, 20\% glycerol, $2 \%$ SDS, $0.5 \%$ (w/v) bromophenol blue), reduced in the presence of $4 \mathrm{mM}$ DTT for $1 \mathrm{~h}$ at room temperature and then alkylated with $10 \mathrm{mM}$ phenylmaleimide for $1 \mathrm{~h}$ at room temperature. A total of $125 \mu \mathrm{g}$ of total protein from each sample were separated by SDS-PAGE on 15\% acrylamide gel, which was stained with Coomassie blue. Each lane was cut into 20 slices and treated with trypsin (Promega). Tryptic peptides were extracted with $60 \%$ acetonitrile and $0.1 \%$ formic acid, evaporated to dryness and resuspended in $25 \mu \mathrm{l}$ of $3 \%$ acetonitrile and $0.1 \%$ formic acid.

Peptide digests were analyzed in duplicate by LC-MS/MS consisting of an Agilent 1100 Series nanoflow liquid chromatography system and a 1100 Series LC/MSD-Trap-SL ion trap mass spectrometer (Agilent Technologies). The peptides were enriched in a Zorbax 300SB-C18 trap column $(5 \mu \mathrm{m}, 5 \times 0.3 \mathrm{~mm})$ and separated by reverse phase chromatography in a Zorbax 300SB-C18 analytical column (Mississauga, Ontario, Canada) $(3.5 \mu \mathrm{m}, 150 \times 0.075 \mathrm{~mm})$ with a gradient of $5-90 \%$ acetonitrile in $0.1 \%$ formic acid at a flow rate of $300 \mathrm{nl} / \mathrm{min}$. The acquisition parameters were as follows: (a) positive mode, (b) scan range from 400 to $2200 \mathrm{Da}$, (c) 'trap drive' of 90 , (d) maximum $3 \mathrm{MS} / \mathrm{MS}$ per cycle, (e) preferred charge state of +2 , (f) active exclusion after two spectra for 1 min and (g) scan speed of $13000 \mathrm{~m} /$ $\mathrm{z} / \mathrm{sec}$. Spectra were recorded in profile mode.

The mass spectra were interpreted and quantified by Spectrum Mill software (Rev. A.03.02.060a, Agilent Technologies). Using a 'Data Extractor' module of the program, the MS/MS spectra were: (a) converted to a centroid mode based on the area in the top $50 \%$ of peak intensity, (b) merged by similarity (for two spectra to be merged, at least 25 out of their 50 most intensive peaks had to match and the matching peaks had to represent $>70 \%$ of the total spectral intensity) within a time window of $\pm 40 \mathrm{~s}$ and $\mathrm{m} / \mathrm{z}$ window $\pm 1.2 \mathrm{Da}$, and (c) filtered by quality (precursor signal to noise ratio $>25$, sequence tag length $>1$ ). For every spectrum, MS intensity was calculated as the area under the extracted chromatogram of ion intensity versus retention time (EIC) employing the same parameters as for merging. 'MS/MS search' was performed for the 25 most intensive peaks in every spectrum with the human UniProt database (7 September, 2006 release: $\mathrm{ftp}: / /$ ftp.expasy.org).

Immunoblotting and reagents. Protein extracts were separated on $12 \%$ SDS-PAGE, as described previously, ${ }^{3-8}$ and probed with monoclonal antibodies against: procaspase-3 (Bio Vision Research Products, Mountain View, CA, USA), activated caspase-3 (Cell Signalling Technology, Pickering, Ontario, Canada), activated caspase-9 (Calbiochem, San Diego, CA, USA), TCTP (Santa Cruz Biotechnology, Santa Cruz, CA, USA), p53 (Santa Cruz Biotechnology), actin (Cedarlane, Burlington, Ontario, Canada), TSG 101 (Abcam, Cambridge, MA, USA), Grp96 (Abcam), tubulin (Oncogene, Boston, MA, USA), phospho-ERK 1/2 (New England Biolabs, Pickering, Ontario, Canada), ERK2 (Santa Cruz Biotechnology), PARP (Cell Signaling) and LC-3 (Abnova Antibody Innovation, Walnut, CA, USA). Protein extracts from the supernatants were TCA precipitated $9: 1$, washed with cold acetone and solubilized in sample buffer. MMC, rapamycin, TCEP (tris (2-carboxyethyl) phosphine) and recombinant PARP were from Sigma (Oakville, Ontario, Canada). Recombinant GST-TCTP and GST were obtained from Abnova Antibody Innovation. LEHD-FMK was from R\&D Systems and PD98059 from Calbiochem. Activated recombinant caspase-3 was obtained from Enzo Life Sciences (Plymouth Meeting, PA, USA).

Fluorescence microscopy for quantization of cells with chromatin condensation and cell membrane permeabilization. Fluorescence microscopy of unfixed/unpermeabilized adherent cells stained with Hoechst 33342 (2' -(4-ethoxyphenyl)-5-(4-methyl-1-piperazinyl)-2.5'-bi$1 \mathrm{H}$-benzimidazole) $(\mathrm{HO})$ and $\mathrm{PI}$ was undertaken as described previously. ${ }^{3-5}$ In brief, cells were grown to confluence in 24-well polycarbonate culture plates (BectonDickinson). $\mathrm{HO}(1 \mu \mathrm{g} / \mathrm{ml})$ was added for $10 \mathrm{~min}$ at $37^{\circ} \mathrm{C}$, and the cells were washed with PBS. PI was added to a final concentration of $5 \mu \mathrm{g} / \mathrm{ml}$ immediately before fluorescence microscopy analysis (excitation filter $I=360-425 \mathrm{~nm}$ ). The percentages of normal, apoptotic and necrotic cells adherent to the dishes were estimated by an investigator blinded to the experimental conditions. Apoptotic cells will show increased $\mathrm{HO}$ fluorescence in absence of PI positivity. Secondary and primary necrotic cells show PI positivity.

Mitochondrial permeability assessment. Loss of mitochondrial permeability of EC was assessed by JC-1 (Molecular Probes, Invitrogen, Burlington, Ontario, Canada) staining and flow cytometry. ${ }^{28}$ Cells were incubated with JC-1 $(10 \mu \mathrm{g} / \mathrm{ml})$ for $15 \mathrm{~min}$, harvested by trypsinization and washed twice with cold PBS. Loss of mitochondrial permeability is indicated by shifting in fluorescence emission from green to red, which was evaluated by flow cytometry (Coulter EPICS XL-MLC flow cytometer, Mississauga, Ontario, Canada) in FL1 and FL2 channels. A total of 10000 events per sample were acquired and the results expressed as fold increase of EC, with loss of mitochondrial permeability relative to control EC 
exposed to normal medium for $4 \mathrm{~h}$ (percentage of experimental cells with red fluorescence/percentage of control cells with red fluorescence).

LDH release assay. LDH release was analyzed by Cyto Tox 96 nonradioactive cytotoxicity assay (Promega), as described by the manufacturer.The results are expressed as the percentage of absorbance at $490 \mathrm{~nm}$ of experimental conditions versus absorbance at $490 \mathrm{~nm}$ of the positive control for necrosis (heating).

Acridine orange staining. Acidic vesicular organelles, which correlate with autophagic activity, were assessed as described previously. ${ }^{29}$ In acridine orange-stained cells, the cytoplasm and nucleolus fluoresce bright green and dim red, whereas acidic compartments fluoresce bright red, evaluated by fluorescence microscopy (excitation filter $1=525-625 \mathrm{~nm}$ ).

Purification of nanovesicles by differential centrifugation. Nanovesicles were prepared from HUVEC and VSMC supernatants by differential centrifugation, as outlined elsewhere ${ }^{30}$ with some modifications. Conditioned media were centrifuged sequentially at $1200 \times g$ for $20 \mathrm{~min}$ to remove cell debris, $50000 \times g$ for $15 \mathrm{~min}$ to eliminate apoptotic blebs and $100000 \times g$ for $18 \mathrm{~h}$ (Beckman SW-41 Ti rotor, Mississauga, Ontario, Canada) to purify the exosomal fraction. For western blot analysis, exosomes were harvested in phosphate buffer $(0.1 \mathrm{M})$, precipitated with TCA $9: 1$, washed with cold acetone and solubilized in SDS-PAGE sample buffer (Bio Rad, Mississauga, Ontario, Canada).

Electron microscopy. For immunogold labeling, nanovesicles isolated as above were adsorbed on parlodium-carbon-coated electron microscopy grids and fixed with $4 \%$ paraformaldehyde. After washing, the grids were floated on ovalbumin $1 \%$ for $5 \mathrm{~min}$ and anti-TCTP antibody (1:20 dilution) for $2 \mathrm{~h}$ at room temperature, washed with PBS, transferred to protein A-gold $(10 \mathrm{~nm})$, washed with PBS and MilliQ water (Billerica, MA, USA), and dried. Uranyl acetate and phosphotungstic acid-negative staining was undertaken, and images were recorded with a Phillips 410 transmission electron microscope (Montréal, Québec, Canada). HUVEC were fixed with paraformaldehyde lysine periodate, dehydrated in increasing methanol solutions and embedded in Lowicryl K4M (Canemco, Lakefield, Qc, Canada) at $-20{ }^{\circ} \mathrm{C}$. For immunocytochemical detection of TCTP, ultrathin sections were incubated in saturated sodium metaperiodate for $8 \mathrm{~min}$, washed with MilliQ water, quenched with ovalbumin $1 \%$ and labeled with anti-TCTP $(1: 10$ dilution) for $4 \mathrm{~h}$ at room temperature, followed by protein A-gold ( 5 or $10 \mathrm{~nm})$. The tissues were stained with uranyl acetate and lead citrate. ${ }^{31}$ For quantification of volume density of MVB and autophagosomes, EC were fixed in glutaraldehyde, postfixed in osmium tetroxyde and embedded in Epon. Electron micrograph fields of the cell cytoplasm were recorded at $\times 7000$ and enlarged at $\times 21000$. The volume density of the MVB and the autophagosomes was evaluated in relation to the cell cytoplasm (nuclei were not included in the evaluation) by direct planimetry using the Zeiss Videopan software (Toronto, Ontario, Canada) (MVB area/cell area $\mu \mathrm{m}^{2}$ ).

Caspase-3 and TCTP silencing. EC were grown in 6- and 24-well plates until $90 \%$ confluence per well. Transfection was performed with Oligofectamine (Invitrogen Life Technologies, Burlington, Ontario, Canada) using ON-TARGET plus SMART pool (Dharmacon, Nepean, Ontario, Canada) for caspase-3, TCTP and control siRNAs. SiRNAs were used at a final concentration of $50 \mathrm{nM}$ (caspase-3) and $200 \mathrm{nM}$ (TCTP) of annealed oligo for $16 \mathrm{~h}$, changed in normal medium for $72 \mathrm{~h}$ (caspase-3) and $48 \mathrm{~h}$ (TCTP) and serum starved for $4 \mathrm{~h}$.

Statistical analysis. The data, expressed as mean \pm S.E.M., were analyzed by Student's $T$-test (with Bonferroni correction when appropriate) or ANOVA, as appropriate.

\section{Conflict of interest}

The authors declare no conflict of interest.

Acknowledgements. This work was supported by research grants from the Canadian Institutes of Health Research to MJH (MOP-15447) and the Canadian Institutes of Health Research to AVP (MOP-66980). MJH is the holder of the Shire Chair in Nephrology, Transplantation and Renal Regeneration of the University of Montreal. IS is the recipient of a training fellowship from the Canadian Institutes of
Health Research. JFC is an FRSQ scholar. We thank the J-L Lévesque Foundation for renewed support, Nicolas Parent for help with JC-1 staining assay and D. Gingras for processing cells for electron microscopy.

1. Lauber K, Bohn E, Krober SM, Xiao YJ, Blumenthal SG, Lindemann RK et al. Apoptotic cells induce migration of phagocytes via caspase-3-mediated release of a lipid attraction signal. Cell 2003; 113: 717-730.

2. Bournazou I, Pound JD, Duffin R, Bournazos S, Melville LA, Brown SB et al. Apoptotic human cells inhibit migration of granulocytes via release of lactoferrin. J Clin Invest 2009; 119: $20-32$.

3. Raymond MA, Desormeaux A, Laplante P, Vigneault N, Filep JG, Landry K et al. Apoptosis of endothelial cells triggers a caspase-dependent anti-apoptotic paracrine loop active on VSMC. Faseb J 2004; 18: 705-707.

4. Laplante P, Raymond MA, Gagnon G, Vigneault N, Sasseville AM, Langelier Y et al. Novel fibrogenic pathways are activated in response to endothelial apoptosis: implications in the pathophysiology of systemic sclerosis. J Immunol 2005; 174: 5740-5749.

5. Laplante P, Raymond MA, Labelle A, Abe J, lozzo RV, Hebert MJ. Perlecan proteolysis induces an alpha2beta1 integrin- and Src family kinase-dependent anti-apoptotic pathway in fibroblasts in the absence of focal adhesion kinase activation. J Biol Chem 2006; 281 : 30383-30392.

6. Cailhier JF, Sirois I, Laplante P, Lepage S, Raymond MA, Brassard N et al. Caspase-3 activation triggers extracellular cathepsin $\mathrm{L}$ release and endorepellin proteolysis. $J$ Biol Chem 2008; 283: 27220-27229.

7. Soulez M, Sirois I, Brassard N, Raymond MA, Nicodeme F, Noiseux N et al. Epidermal growth factor and perlecan fragments produced by apoptotic endothelial cells co-ordinately activate ERK1/2-dependent antiapoptotic pathways in mesenchymal stem cells. Stem Cells 2010; 28: 810-820.

8. Laplante P, Sirois I, Raymond MA, Kokta V, Beliveau A, Prat A et al. Caspase-3-mediated secretion of connective tissue growth factor by apoptotic endothelial cells promotes fibrosis. Cell Death Differ 2010; 17: 291-303.

9. Cailhier JF, Laplante P, Hebert MJ. Endothelial apoptosis and chronic transplant vasculopathy: recent results, novel mechanisms. Am J Transplant 2006; 6: 247-253.

10. Cornell LD, Smith RN, Colvin RB. Kidney transplantation: mechanisms of rejection and acceptance. Annu Rev Pathol 2008; 3: 189-220.

11. Pollman MJ, Hall JL, Mann MJ, Zhang L, Gibbons GH. Inhibition of neointimal cell bcl-x expression induces apoptosis and regression of vascular disease. Nat Med 1998; 4 : 222-227.

12. Gennaro G, Menard C, Michaud SE, Deblois D, Rivard A. Inhibition of vascular smooth muscle cell proliferation and neointimal formation in injured arteries by a novel, oral mitogen-activated protein kinase/extracellular signal-regulated kinase inhibitor. Circulation 2004; 110: 3367-3371.

13. Hirata A, Igarashi $M$, Yamaguchi $H$, Suwabe A, Daimon $M$, Kato $T$ et al Nifedipine suppresses neointimal thickening by its inhibitory effect on vascular smooth muscle cell growth via a MEK-ERK pathway coupling with Pyk2. Br J Pharmacol 2000; 131: 1521-1530.

14. Pshezhetsky AV, Fedjaev M, Ashmarina L, Mazur A, Budman L, Sinnett D et al. Subcellular proteomics of cell differentiation: quantitative analysis of the plasma membrane proteome of Caco-2 cells. Proteomics 2007; 7: 2201-2215.

15. Thery $\mathrm{C}$, Ostrowski M, Segura E. Membrane vesicles as conveyors of immune responses. Nat Rev Immunol 2009; 9: 581-593.

16. Amzallag N, Passer BJ, Allanic D, Segura E, Thery C, Goud B et al. TSAP6 facilitates the secretion of translationally controlled tumor protein/histamine-releasing factor via a nonclassical pathway. J Biol Chem 2004; 279: 46104-46112.

17. Yu X, Harris SL, Levine AJ. The regulation of exosome secretion: a novel function of the p53 protein. Cancer Res 2006; 66: 4795-4801.

18. Lespagnol A, Duflaut D, Beekman C, Blanc L, Fiucci G, Marine JC et al. Exosome secretion, including the DNA damage-induced p53-dependent secretory pathway, is severely compromised in TSAP6/Steap3-null mice. Cell Death Differ 2008; 15: 1723-1733.

19. Yoon T, Jung J, Kim M, Lee KM, Choi EC, Lee K. Identification of the self-interaction of rat TCTP/lgE-dependent histamine-releasing factor using yeast two-hybrid system. Arch Biochem Biophys 2000; 384: 379-382.

20. Kim M, Min HJ, Won HY, Park H, Lee JC, Park HW et al. Dimerization of translationally controlled tumor protein is essential for its cytokine-like activity. PLOS ONE 2009; 4: e6464.

21. Thery C, Boussac M, Veron P, Ricciardi-Castagnoli P, Raposo G, Garin J et al. Proteomic analysis of dendritic cell-derived exosomes: a secreted subcellular compartment distinct from apoptotic vesicles. J Immunol 2001; 166: 7309-7318.

22. Thery C, Zitvogel L, Amigorena S. Exosomes: composition, biogenesis and function Nat Rev Immunol 2002; 2: 569-579.

23. Li XB, Zhang ZR, Schluesener HJ, Xu SQ. Role of exosomes in immune regulation. J Cell Mol Med 2006; 10: 364-375.

24. Kroemer G, Galluzzi L, Vandenabeele P, Abrams J, Alnemri ES, Baehrecke EH et al. Classification of cell death: recommendations of the Nomenclature Committee on Cell Death 2009. Cell Death Differ 2009; 16: 3-11.

25. Chen SH, Wu PS, Chou CH, Yan YT, Liu H, Weng SY et al. A knockout mouse approach reveals that TCTP functions as an essential factor for cell proliferation and survival in a tissue- or cell type-specific manner. Mol Biol Cell 2007; 18: 2525-2532. 
26. Telerman A, Amson R. The molecular programme of tumour reversion: the steps beyond malignant transformation. Nat Rev Cancer 2009; 9: 206-216.

27. Vonakis BM, Macglashan Jr DW, Vilarino N, Langdon JM, Scott RS, MacDonald SM. Distinct characteristics of signal transduction events by histamine-releasing factor/ translationally controlled tumor protein (HRF/TCTP)-induced priming and activation of human basophils. Blood 2008; 111: 1789-1796.

28. Paquet C, Sane AT, Beauchemin M, Bertrand R. Caspase- and mitochondrial dysfunctiondependent mechanisms of lysosomal leakage and cathepsin B activation in DNA damage-induced apoptosis. Leukemia 2005; 19: 784-791.

29. Takeuchi H, Kanzawa T, Kondo Y, Kondo S. Inhibition of platelet-derived growth factor signalling induces autophagy in malignant glioma cells. Br J Cancer 2004; 90: 1069-1075.

30. Raposo G, Nijman HW, Stoorvogel W, Liejendekker R, Harding CV, Melief CJ et al. B lymphocytes secrete antigen-presenting vesicles. J Exp Med 1996; 183: 1161-1172.

31. Bendayan M. Tech.Sight. Worth its weight in gold. Science 2001; 291: 1363-1365.

32. Pisitkun T, Shen RF, Knepper MA. Identification and proteomic profiling of exosomes in human urine. Proc Natl Acad Sci USA 2004; 101: 13368-13373.

33. Gutwein P, Stoeck A, Riedle S, Gast D, Runz S, Condon TP et al. Cleavage of L1 in exosomes and apoptotic membrane vesicles released from ovarian carcinoma cells. Clin Cancer Res 2005; 11: 2492-2501.

34. Wubbolts R, Leckie RS, Veenhuizen PT, Schwarzmann G, Mobius W, Hoernschemeyer J et al. Proteomic and biochemical analyses of human B cell-derived exosomes. Potential implications for their function and multivesicular body formation. J Biol Chem 2003; 278 : 10963-10972.

35. Aronov S, Gelin-Licht R, Zipor G, Haim L, Safran E, Gerst JE. mRNAs encoding polarity and exocytosis factors are cotransported with the cortical endoplasmic reticulum to the incipient bud in Saccharomyces cerevisiae. Mol Cell Biol 2007; 27: 3441-3455.

36. Nguyen NV, Gleeson PA, Courtois-Coutry N, Caplan MJ, Van Driel IR. Gastric parietal cell acid secretion in mice can be regulated independently of $\mathrm{H} / \mathrm{K}$ ATPase endocytosis. Gastroenterology 2004; 127: 145-154.

37. Wang P, Tortorella M, England K, Malfait AM, Thomas G, Arner EC et al. Proprotein convertase furin interacts with and cleaves pro-ADAMTS4 (Aggrecanase-1) in the trans-Golgi network. J Biol Chem 2004; 279: 15434-15440.

38. Emeis JJ, van den Eijnden-Schrauwen $Y$, van den Hoogen $\mathrm{CM}$, de Priester W, Westmuckett A, Lupu F. An endothelial storage granule for tissue-type plasminogen activator. J Cell Biol 1997; 139: 245-256.

39. van Niel G, Raposo G, Candalh C, Boussac M, Hershberg R, Cerf-Bensussan N et al. Intestinal epithelial cells secrete exosome-like vesicles. Gastroenterology 2001; 121: 337-349.

40. Segura E, Amigorena S, Thery C. Mature dendritic cells secrete exosomes with strong ability to induce antigen-specific effector immune responses. Blood Cells Mol Dis 2005; 35: 89-93.

\section{Supplementary Information accompanies the paper on Cell Death and Differentiation website (http://www.nature.com/cdd)}

